See discussions, stats, and author profiles for this publication at: https://www.researchgate.net/publication/333401727

\title{
An annotated check list of the Cicadomorpha and Fulgoromorpha (Hemiptera) of the Madeira and Salvages archipelagos
}

Article in Zootaxa $\cdot$ May 2008

DOI: 10.11646/20otaxa.1762.1.1.

CITATIONS

5

2 authors, including:

Dora Pombo

Universidade da Madeira

74 PUBLICATIONS 320 CITATIONS

SEE PROFILE

Some of the authors of this publication are also working on these related projects:

Estudo da aplicabilidade da luta biotecnica no control do bichado da castanha, Cydia splendana, na Madeira View project

Comparative cytogenetics of Insecta View project 


\title{
An annotated check list of the Cicadomorpha and Fulgoromorpha (Hemiptera) of the Madeira and Salvages archipelagos
}

\author{
DORA AGUIN-POMBO ${ }^{1,2}$, CARLOS FREITAS ${ }^{2}$ \\ 'Department of Biology, University of Madeira, Campus da Penteada, 9000-390 Funchal, Madeira, Portugal, e-mail: aguin@uma.pt \\ ${ }_{2}^{2}$ CEM, Centre for Macaronesian Studies, Campus da Penteada, 9000-390 Funchal, Madeira, Portugal
}

\section{TABLE OF CONTENTS}

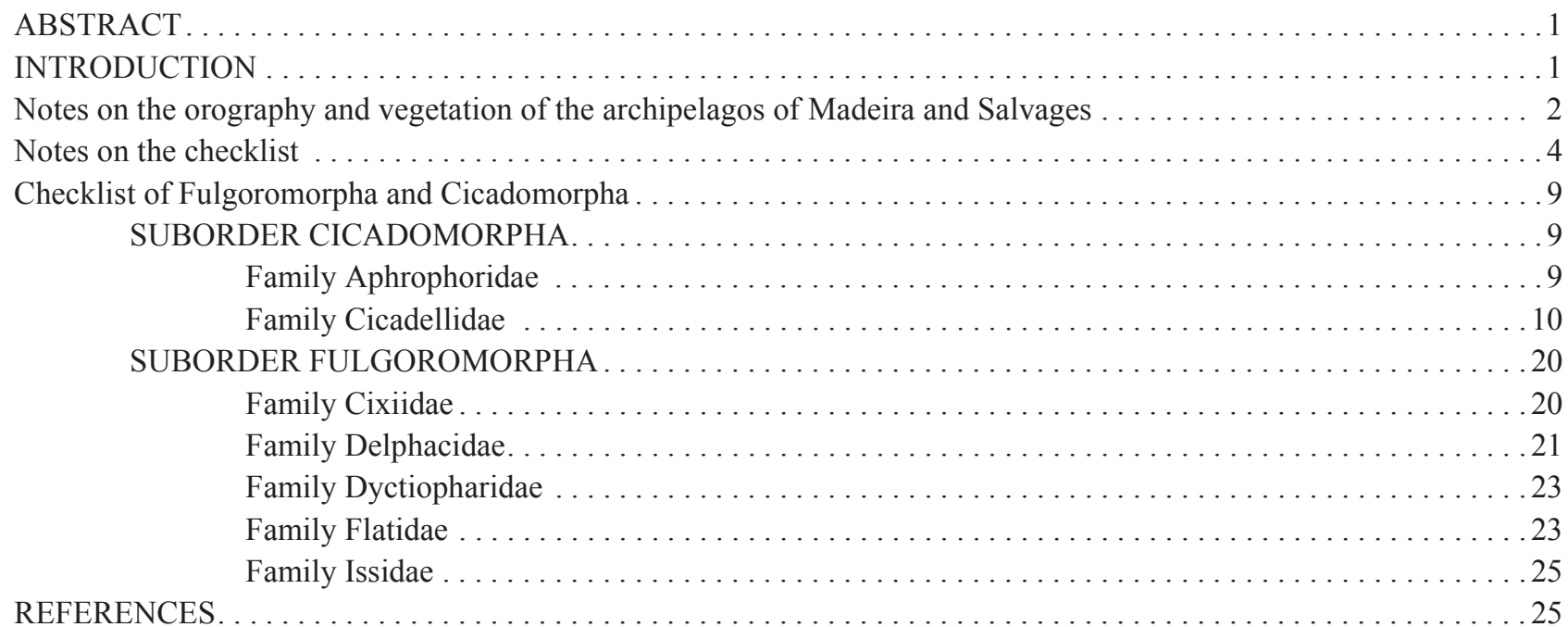

\begin{abstract}
The volcanic archipelagos of Madeira and Salvages located respectively at $635 \mathrm{~km}$ and $373 \mathrm{~km}$ offshore of the northeastern African coast are very rich in endemic species. These two archipelagos together with the Canary Ils and the archipelagos of Cape Verde and Azores represent part of the Mediterranean hot spot which is considered the richest area in flora and fauna of Europe. To synthesize the knowledge on these groups, an annotated checklist of Cicadomorpha and Fulgoromorpha of the archipelagos of Salvages and Madeira is presented based on published literature including data on distribution and food plants. In addition 9 new species records are reported: Austragallia caboverdensis, Austragallia sinuata, Balclutha rufofascia, B. hebe, Cicadella viridis, Macrosteles sexnotatus, Recilia angusticeps, Tamaricella cf fasciolata and Zyginidia lineata. The checklist comprises 79 species of 7 different families. Of these species $87 \%$ occur in Madeira and $10 \%$ in the Salvages archipelago. As a whole 27\% are endemic to Madeira and $8 \%$ are endemic to Macaronesia. Despite this diversity, knowledge of their habitats and food plants is still very scarce.
\end{abstract}

KEY WORDS: Homoptera, Auchenorrhyncha, distribution, new records, Madeira, Hemiptera, Macaronesia

\section{INTRODUCTION}

Macaronesian archipelagos are included in the Mediterranean hot spot which is the richest area in flora and fauna of the European Union (Médail and Quézel, 1999). The Macaronesia subregion located between Eurasia 
and Africa includes parts of the west coast of Africa (Morocco and Mauritania) and five archipelagos of volcanic origin: Madeira, Azores, Salvages, Canary Islands and Cape Verde. The archipelagos of Madeira and Salvages although are small in surface $\left(\sim 800 \mathrm{~km}^{2}\right)$ have an endemicity rate considerably high for oceanic islands, namely for land snails, Madeira shows among the largest number of endemic species per squared kilometre in the World, similar to other oceanic islands as Hawaii or Maurice Islands (Waldén, 1993). However, insular taxa show usually less genetic variability and are more prone of extinction than continental ones (Frankham, 1997; Vitousek, 1988) and these archipelagos are not an exception. In the last decades, major environmental modifications have occurred related to an increase in tourism pressure. Tourism represents for Madeira an opportunity for economic development but is also a threat to these fragile ecosystems. Madeira has a high population density with approximately 330 inhabitants per squared kilometre, the largest in Portugal. Thus, the intensive construction of houses, hotels and roads together with the threat of an increasing number of invasive species arriving through external commercial exchanges (Aguin-Pombo et al. 2007; Freitas and Aguin-Pombo, 2004; Wetterer et al. 2006), requires the implementation of conservation strategies for the native flora and fauna. To address planning and conservation issues, the first step is to have valid species checklists. In the neighboring archipelagos of Canary Isls, Azores and Cape Verde, a considerable effort has recently been made to compile checklists of all terrestrial taxa (Aguin-Pombo et al. 2005; Oromi et al. 2004, Quartau, 1993, Quartau and Borges, 2005), yet no recent similar checklists have been published for Madeira.

Cicadomorpha and Fulgoromorpha are not very diverse in the Macaronesia archipelagos but some genera like Asianidia Zachvatkin 1946, Cyphopterum Melichar, 1905, Issus Fabricius, 1803 and Cixius Latreille, 1804 have undergone remarkable radiations resulting in many endemic species and in a genus endemic to Madeira and the Canary Islands, Makarorysa Remane \& Asche, 1986. Concerning Cicadomorpha, a checklist of Madeira species was published some years ago (Quartau, 1993) but in recent decades nomenclature changes, corrections of misidentifications, reports of new records and revisions of several genera, have added new information for species of this area. This work updates the published knowledge on Cicadomorpha and Fulgoromorpha for the archipelagos of Madeira and Salvages. Information on distribution and food plants and/or habitat for species of both groups has been compiled and nine new species records to Madeira fauna are reported. Clearly the results of this work accentuate the disharmonic character of the fauna of these oceanic islands. Especially remarkable is the absence of Cicadoidea from all Macaronesian archipelagos, particularly considering that cicadas are well represented in the nearby regions of the Iberian Peninsula and North Africa. Other common continental families absent from the Macaronesian archipelagos were Membracidae and Ulopidae. Madeira also lacks representatives of some families which are present in the Canary Islands like Kinnaridae, Tettigometridae, Meenoplidae and Tropiduchidae. The isolation of Madeira from the mainland may account for these differences. Although some information has been published on host plant and habitat associations for species of Madeira, more field work on this group is clearly needed.

\section{Notes on the orography and vegetation of the Madeira and Salvages archipelagos}

The Madeira archipelago is closer to the north-western African coast ( $\sim 635 \mathrm{~km})$ than to Europe (794 km from Portugal) and geologically isolated from the Salvages archipelago which is located only $175 \mathrm{~km}$ from the Canary Islands and $373 \mathrm{~km}$ from the African coast. These archipelagos consist of several oceanic islands and islets of volcanic origin ocupying a surface of only about $800 \mathrm{~km}^{2}$ (Fig. 1). Madeira comprises two main inhabited islands, Madeira and Porto Santo, and three islets known as the Desertas Islands -Ilhéu do Chão, Deserta Grande and Deserta Pequena or Bugio- and about 10 offshore rocks. The island of Madeira represents almost $93 \%$ of the land surface and it is very steep with about $90 \%$ of its surface above $500 \mathrm{~m}$. In contrast, Porto Santo, located $42 \mathrm{~km}$ further north, and the Desertas Islands, about $24 \mathrm{~km}$ off the southeastern coast of Madeira, are rather flat, with their highest altitudes below $520 \mathrm{~m}$. 


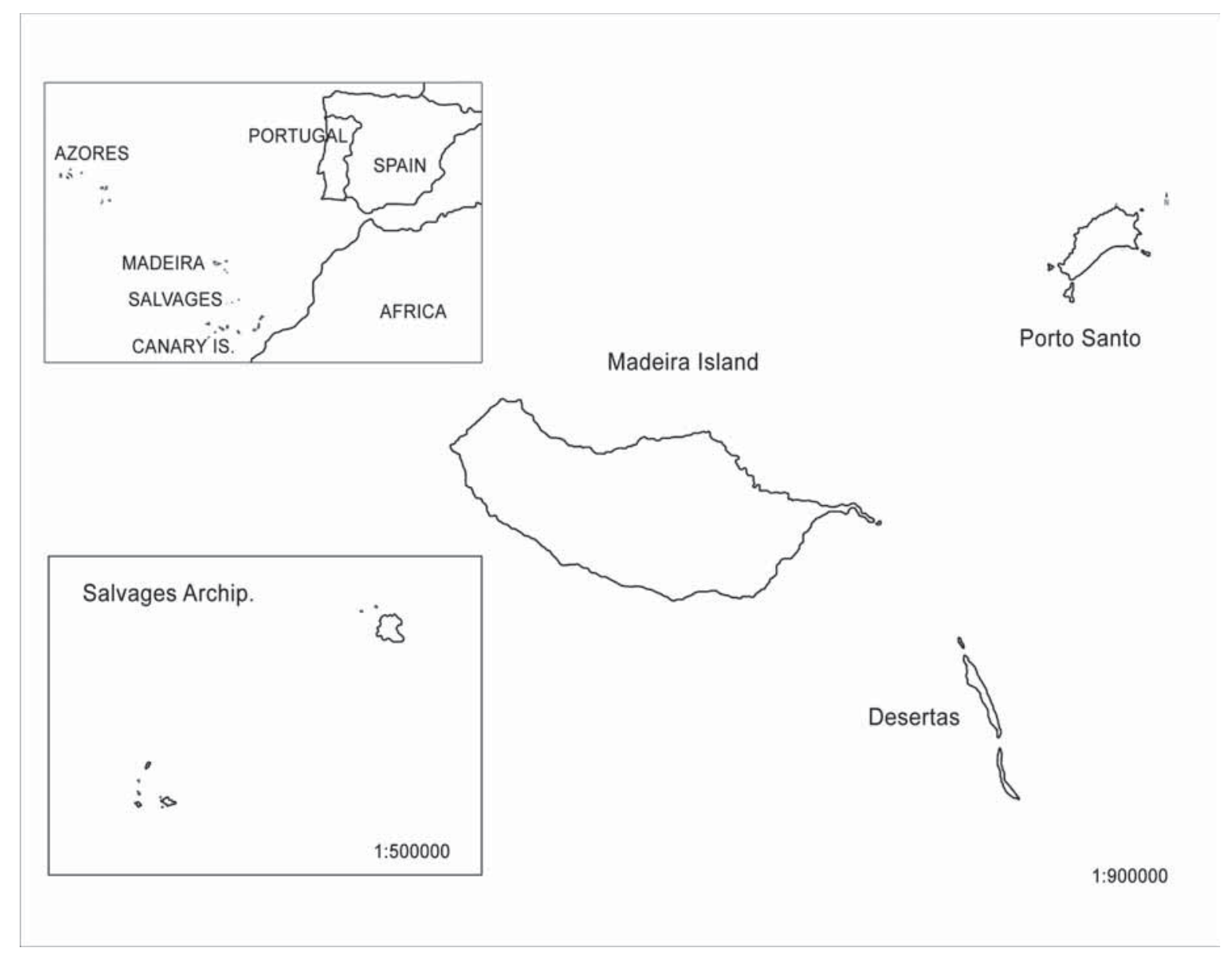

FIGURE 1. Location of the archipelagos of Madeira and Salvages and their relative location to the Iberian Peninsula, north of Africa and Azores archipelagos.

The islands of the Madeira archipelago also differ ecologically. Madeira Island is the largest and the highest $(1862 \mathrm{~m})$ of all and also the most diverse in habitats. It shows a clear north-south differentiation due to different sun exposure, humidity and annual median temperature. The most characteristic vegetation is the coastal vegetation and the evergreen laurel forest (dry and wet) growing at medium altitudes which is considered a remnant of the Tertiary Age (Press and Short, 1994). The dry evergreen forest is much reduced and grows in areas with high mean temperature and low annual precipitation. This forest has as characteristic tree species Apolonias barbujana, Visnea mocanera and Picconia excelsa. The wet laurel forest grows from 300$700 \mathrm{~m}$ up to $1300 \mathrm{~m}$ in more humid areas with lowest mean annual temperatures and has characteristic hygrophilous tree species of Lauraceae endemic to Macaronesia, such as Laurus novocanariensis, Ocotea foetens and Persea indica. From $1300 \mathrm{~m}$ up to $1500 \mathrm{~m}$ the vegetation consists mainly of shrubs, with Erica arborea and Vaccinium padifolium dominant; above this, only herbaceous vegetation occurs. The eastern part of the Island (Ponta de São Lourenço), is semi-arid with xerophytic vegetation due to the smooth landscape, low annual precipitation and strong winds. Porto Santo is a small, rather flat island, with only $42 \mathrm{square}$ km and $517 \mathrm{~m}$ at its highest point, covered mainly by xeric vegetation. Here there is little differentiation between the southern and northern slopes in climate and plant communities.

The Salvages archipelago, located $174 \mathrm{~km}$ north of the Canaries and $255 \mathrm{~km}$ south of Madeira, consists of three small islets and several rocks covered by halophytic vegetation. The biggest of the three is the Selvagem Grande, and the other two islets are Selvagem Pequena or Pitão Grande, and Ilhéu de Fora or La Salvajita. Selvagem Grande, $\sim 2.45 \mathrm{~km}^{2}$ in size, is flat and about $100 \mathrm{~m}$ above the sea surface with Pico the Atalaia 
$(163 \mathrm{~m})$ as its highest point; Selvagem Pequena is only $\sim 0.3 \mathrm{~km}^{2}$ and $49 \mathrm{~m}$ at its high point while Ilhéu de Fora has $\sim 0.08 \mathrm{~km}^{2}$ and $18 \mathrm{~m}$ at the highest point.

\section{Notes on the checklist}

The classification and information on the general distribution for each species mainly follows Hoch (2007). Families, subfamilies, genera and species of each suborder are arranged alphabetically and new species records are indicated by the symbol $(*)$. The material studied is in the collection of the first author. The distribution of each species in the Madeira and Salvages archipelagoes was compiled according to published records; excluding previous compilations with no original information. When it was necessary to add comments, these were reported under the heading remarks. Additional information on food plants and/or habitats in Madeira has been included (Table 2 and Table 3). A summary of the number of species, and endemics per island is shown in Table 1. Lindberg and China contributed greatly to the knowledge of the fauna of Madeira, however, many errors in identification have been reported especially for morphologically variable species. Therefore, additional material for these species together with further study of material from these previous authors will be necessary before a definitive species checklist is possible.

TABLE 1. Species of Cicadomorpha and Fulgoromorpha indicating the total number of species, endemic species and species per island of each family.

Island abbreviations: Porto Santo Island (PS); Madeira Island (Ma); Ilhéu Chão (IC); Deserta Grande (DG); Bugio (Bu); Selvagem Grande (Sg); Selvagem pequena (Sp); Pitão pequena (Pp).

\begin{tabular}{|c|c|c|c|c|c|c|c|c|c|c|c|}
\hline \multirow[t]{2}{*}{ Suborder } & \multirow[t]{2}{*}{ Family } & \multicolumn{5}{|c|}{ Madeira } & \multicolumn{3}{|c|}{ Selvagens } & \multirow{2}{*}{$\begin{array}{l}\text { Endemic } \\
\text { species }\end{array}$} & \multirow{2}{*}{$\begin{array}{l}\text { Species } \\
\text { number }\end{array}$} \\
\hline & & Ps & Ma & Ic & Dg & Bu & $\mathrm{Sg}$ & Sp & Pp & & \\
\hline \multirow[t]{2}{*}{ Cicadomorpha } & Aphrophoridae & 1 & 1 & & 1 & & & & & 0 & 1 \\
\hline & Cicadellidae & 14 & 46 & 3 & 4 & 1 & 2 & 4 & & 10 & 53 \\
\hline \multirow[t]{5}{*}{ Fulgoromorpha } & Cixiidae & & 4 & 1 & & & & & & 5 & 5 \\
\hline & Delphacidae & 2 & 14 & & & & & & & 2 & 14 \\
\hline & Dictyopharidae & & 1 & & & & & & & 0 & 1 \\
\hline & Flatidae & 1 & 2 & 1 & 1 & & & 2 & & 4 & 4 \\
\hline & Issidae & & 1 & & & & & & & 1 & 1 \\
\hline Total & & 18 & 69 & 5 & 6 & 1 & 2 & 6 & & 22 & 79 \\
\hline$\%$ of total species & & $23 \%$ & $87 \%$ & $6 \%$ & $8 \%$ & $1 \%$ & $2 \%$ & $8 \%$ & & $28 \%$ & \\
\hline
\end{tabular}


TABLE 2. Checklist, distribution of the species of Cicadomorpha and Fulgoromorpha of Madeira and Selvagens indicating the number of known food plants for each species on these archipelagos.

Present on the island (+); dubious record (\#); new record (*). Island abbreviations: Porto Santo Island (PS); Madeira Island (Ma); Ilhéu Chão (IC); Deserta Grande (DG); Bugio (Bu); Selvagem Grande (Sg); Selvagem pequena (Sp); Pitão Pequena (Pp). Archipelagos abreviations: Mac (Macaronésia), Mad (Madeira); Sal (Salvages).

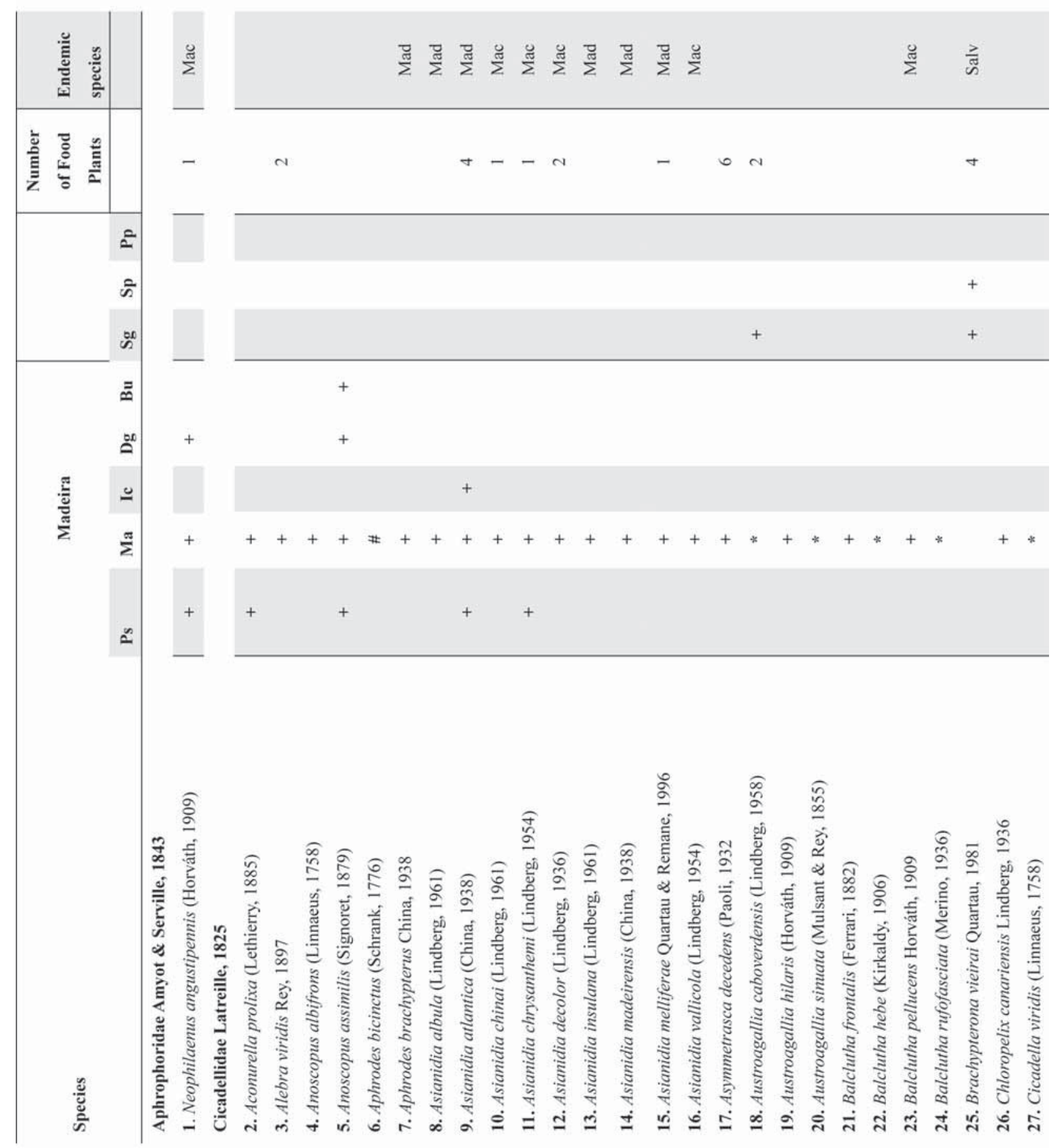


TABLE 2 (continued).

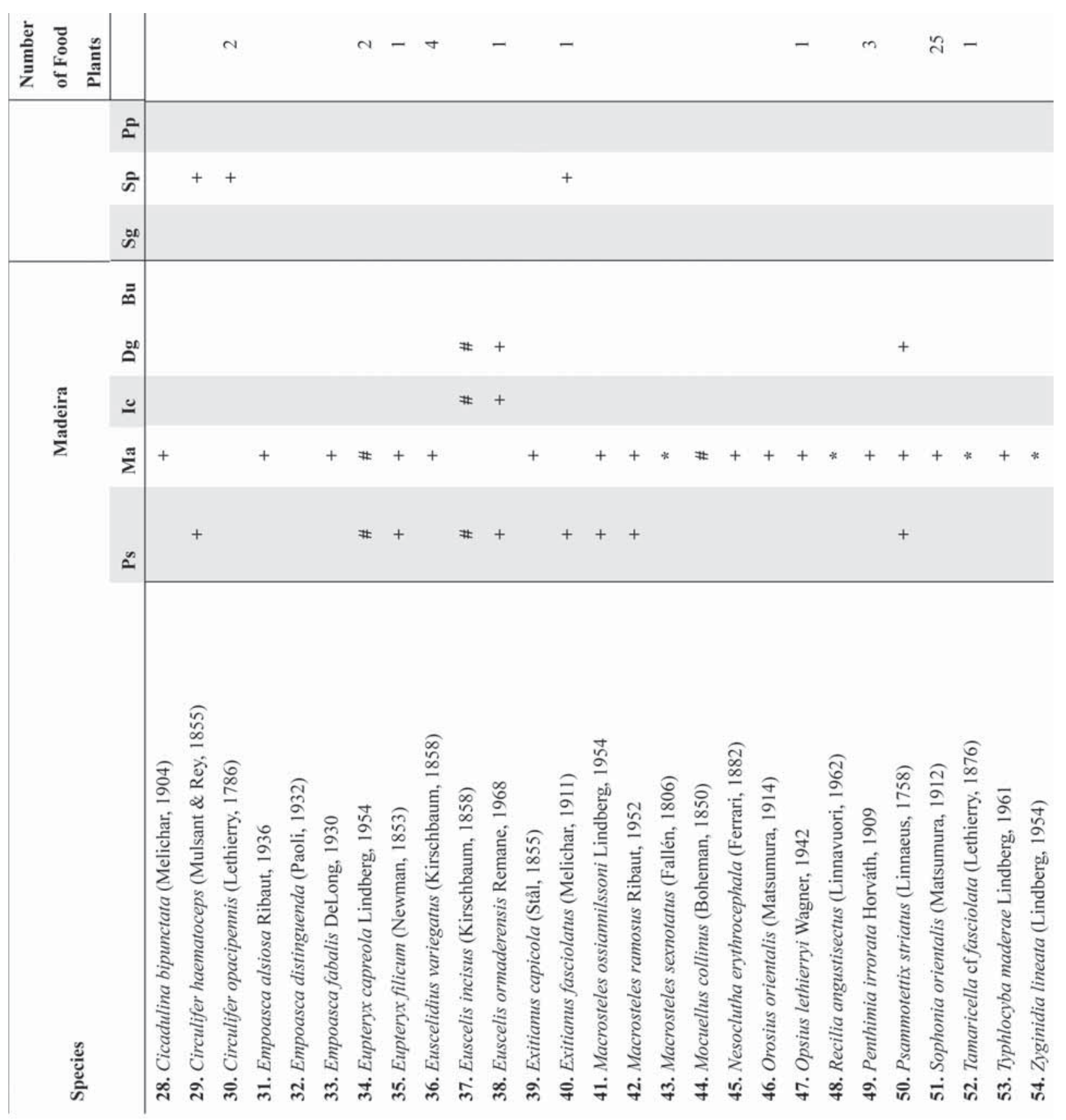

continued on the next page 
TABLE 2 (continued).

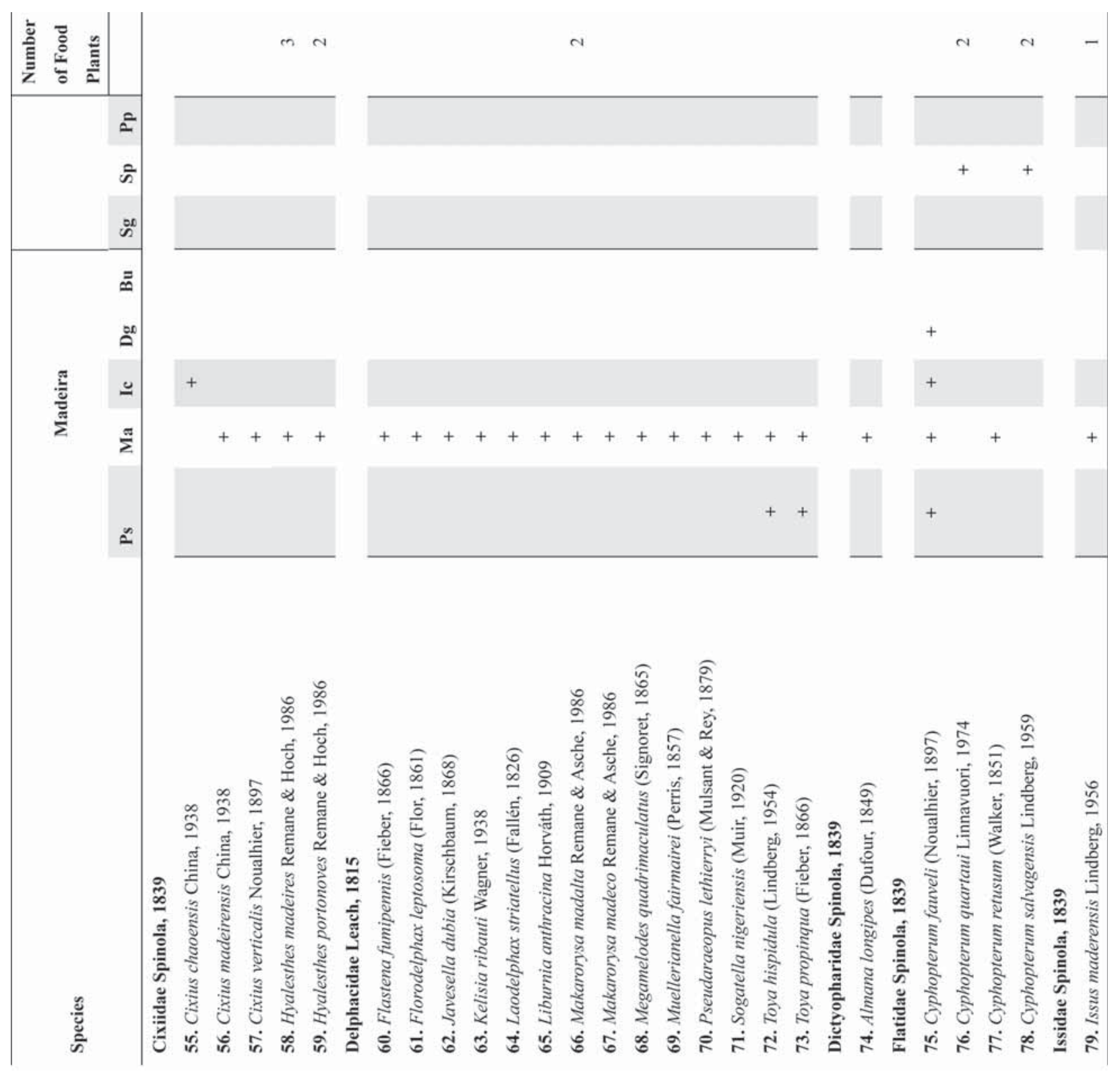


TABLE 3. Food plant species of Cicadomorpha and Fulgoromorpha reported for the archipelagos of Madeira and Salvages indicating the source of information. The insect species are represented by numbers as in Table 2.

\begin{tabular}{|c|c|c|c|}
\hline Families & Food Plants & Insect species & Information on food plants \\
\hline Aizoaceae & 1.Tetragonia tetragonoides & 18 & This work \\
\hline Anacardiaceae & 2.Schinus molle & 17,51 & Freitas \& Aguin-Pombo 2004; Aguin-Pombo et al. 2006 \\
\hline \multirow[t]{8}{*}{ Asteraceae } & 3.Artemisia arborescens & 9 & Quartau \& Remane 1996 \\
\hline & 4.Argyranthemum haematomma & 51 & Aguin-Pombo et al. 2006 \\
\hline & 5.Artemisia argentea & 9,38 & Lindberg, 1961; Remane 1968 \\
\hline & 6.Artemisia canariensis & 9 & Quartau \& Remane 1996 \\
\hline & 7.Argyranthemum pinnatifidum & 9,11 & Lindberg 1961; Quartau \& Remane 1996 \\
\hline & 8.Dahlia sp. & 17 & Freitas \& Aguin-Pombo 2004 \\
\hline & 9.Osteospermum barberae & 51 & Aguin-Pombo et al. 2006 \\
\hline & 10.Taigetes minuta & 12,51 & Freitas \& Aguin-Pombo 2004; Aguin-Pombo et al. 2006 \\
\hline Caricaceae & 11.Carica papaya & 51 & Aguin-Pombo et al. 2006 \\
\hline Chenopodiaceae & 12.Suaeda vera & $25,30,76,78$ & $\begin{array}{l}\text { Leise 1992; Linnavuori 1974; Quartau 1975; Quartau } \\
1981\end{array}$ \\
\hline \multirow[t]{2}{*}{ Convolvulaceae } & 13.Ipomoea batatas & 51 & Aguin-Pombo et al. 2006 \\
\hline & 14.Ipomoea spp & 51 & Aguin-Pombo et al. 2006 \\
\hline Dennstaedtiaceae & 15.Pteridum aquilinum & 34,35 & Lindberg 1961; Quartau \& Fançony 1988 \\
\hline \multirow[t]{2}{*}{ Ericaceae } & 16.Erica spp. & $53,51,66,79$ & $\begin{array}{l}\text { Aguin-Pombo et al. 2006; Lindberg 1961; Remane \& } \\
\text { Asche } 1986\end{array}$ \\
\hline & 17.Vaccinium spp. & 66 & Lindberg 1961; Remane 1968 \\
\hline \multirow[t]{4}{*}{ Euphorbiaceae } & 18.Acalypha wilkesiana & 51 & Aguin-Pombo et al. 2006 \\
\hline & 19.Euphorbia mellifera & 58,59 & Hoch \& Remane 1985; Remane \& Hoch 1986 \\
\hline & 20.Euphorbia spp. & 15 & Quartau \& Remane 1996 \\
\hline & 21.Ricinus communis & 17,51 & Freitas \& Aguin-Pombo 2004; Aguin-Pombo et al. 2006 \\
\hline Fabaceae & 22.Arachnis hypogea & 51 & Aguin-Pombo et al. ,2006 \\
\hline \multirow[t]{2}{*}{ Fagaceae } & 23.Quercus robur & 3 & Aguin-Pombo 2002 \\
\hline & 24.Castanea sativa & 2,3 & Lindberg 1961; Aguin-Pombo 2002 \\
\hline Geraniaceae & 25.Erodium moschatum & 51 & Aguin-Pombo et al. 2006 \\
\hline Globulariaceae & 26.Globularia salicina & 51 & $\begin{array}{l}\text { Hoch \& Remane 1985; Remane \& Hoch 1986; Aguin- } \\
\text { Pombo et al. } 2006\end{array}$ \\
\hline Labiatae & 27.Labiatae spp. & 11 & Lindberg 1961 \\
\hline Lauraceae & 28.Persea americana & 51 & Aguin-Pombo et al. 2006 \\
\hline \multirow[t]{3}{*}{ Leguminosae } & 29.Agropyron junceiforme & $24,30,40,75,78$ & Leise 1992; Quartau 1975, 1984 \\
\hline & 30.Lotus salvagensis & 24 & Leise, 1992 \\
\hline & 31.Trifolium repens & 36 & Freitas \& Aguin-Pombo 2003 \\
\hline \multirow[t]{2}{*}{ Malvaceae } & 32.Hibiscus rosa-sinensis & 51 & Aguin-Pombo et al. 2006 \\
\hline & 33.Lavatera cretica & 17,51 & Freitas \& Aguin-Pombo 2004; Aguin-Pombo et al. 2006 \\
\hline \multirow[t]{2}{*}{ Myrtaceae } & 34.Psidium guajava & 51 & Aguin-Pombo et al. 2006 \\
\hline & 35.Eugenia uniflora & 51 & Aguin-Pombo et al. 2006 \\
\hline \multirow[t]{2}{*}{ Passifloraceae } & 36.Passiflora edulis & 17,49 & Freitas \& Aguin-Pombo 2004; Aguin-Pombo et al. 2006 \\
\hline & 37.Passiflora laurifolia & 49 & Aguin-Pombo et al. 2006 \\
\hline
\end{tabular}


TABLE 3 (continued).

\begin{tabular}{|c|c|c|c|}
\hline Families & Food Plants & Insect species & Information on food plants \\
\hline \multirow[t]{3}{*}{ Poaceae } & 38.Brachiaria mutica & 1 & Quartau \& André 1986 \\
\hline & 39.Brachypodium sylvaticum & 36 & Freitas \& Aguin-Pombo 2004 \\
\hline & 40.Poaceae spp. & 36 & Freitas \& Aguin-Pombo 2004 \\
\hline \multirow[t]{2}{*}{ Polygonaceae } & 41.Rumex maderensis & 51 & Aguin-Pombo et al. 2006 \\
\hline & 42.Rumex spp. & 49 & Aguin-Pombo et al. 2006 \\
\hline Pteridaceae & 43.Pteridium aquilinum & 34 & Quartau \& Fançony 1988 \\
\hline Rosaceae & 44.Rubus spp. & 58 & Hoch \& Remane 1985; Remane \& Hoch 1986 \\
\hline \multirow[t]{2}{*}{ Rutaceae } & 45.Casimiroa edulis & 52 & Aguin-Pombo et al. 2006 \\
\hline & 46.Citrus sinensis & 52 & Aguin-Pombo et al. 2006 \\
\hline Scrophulariaceae & 47.Isoplexis sceptrum & 10 & Quartau et al. 2004 \\
\hline \multirow[t]{3}{*}{ Solanaceae } & 48.Cyphomandra betacea & 52 & Aguin-Pombo et al. 2006 \\
\hline & 49.Datura stramonium & 52 & Aguin-Pombo et al. 2006 \\
\hline & 50.Lycopersicon esculentum & 18,24 & Quartau 1981; Quartau \& André 1990; this work \\
\hline Tamaricaceae & 51.Tamarix spp. & 47,52 & Lindberg 1961; Leise 1992; this work \\
\hline Umbeliferae & 52.Apium nodiflorum & 36 & Freitas \& Aguin-Pombo 2004 \\
\hline \multirow[t]{2}{*}{ Verbenaceae } & 53.Echium spp. & 58,59 & Remane \& Hoch 1986; Hoch \& Remane 1985 \\
\hline & 54.Enterolobium cyclocarpium & 17 & Freitas \& Aguin-Pombo 2004 \\
\hline Vitaceae & 55.Vitis vinifera & 51 & Aguin-Pombo et al. 2006 \\
\hline Zingiberaceae & 56.Hedychium gardneranum & 51 & Aguin-Pombo et al. 2006 \\
\hline
\end{tabular}

\section{CHECKLIST OF CICADOMORPHA AND FULGOROMORPHA}

\section{Suborder Cicadomorpha Evans, 1946}

\section{Family Aphrophoridae Amyot \& Serville, 1843}

\section{Neophilaenus angustipennis (Horváth, 1909)}

Philaenus angustipennis Horváth, 1909: 298

Neophilaenus angustipennis; Quartau and André, 1988: 244

Distribution. Endemic to Canary Islands and Madeira archipelago but not listed in recent checklists (Hoch, 2004). DESERTA GRANDE: on plateau (Quartau and André 1988). MADEIRA: recorded mainly from southern, driest part of island (Ponta de São Lourenço), several localities along southern shore, and up to $900 \mathrm{~m}$ altitude (Ribeira da Janela) on northern coast (Quartau and André 1988). PORTO SANTO: recorded from Portela in north (Quartau and André 1988).

Food plants. Found on Gramineae (Quartau and André, 1988).

Remarks. Quartau and André (1988) suggested that this species probably arrived in Madeira between 1971 and 1980. 


\section{Family Cicadellidae Latreille, 1825}

\section{Subfamily Agalliinae Kirkaldy, 1901}

*Austroagallia caboverdensis (Lindberg, 1958)

Peragallia caboverdensis Lindberg, 1958: 195; Quartau and André, 1990: 69

Distribution. Afrotropical region, Cape Verde and Salvages Archipelagos but not listed from latter in recent checklists (Hoch, 2004). MADEIRA: recorded from southern coast (this work). SELVAGEM GRANDE: locality unknown (Quartau and André, 1990).

Food plants. Lycopersicon esculetum (Quartau and André, 1990) and Tetragonia tetragonoides (this work).

MATERIAL EXAMINED: $1 \sigma^{\star}$ São Vicente, Madeira, 10m, 1.x.2000, on Tetragonia tetragonoides, Leg. N. Freitas.

Austroagallia hilaris (Horváth, 1909)

Agallia hilaris Horváth, 1909: 297; China, 1938: 44

Distribution. Canary Islands, Madeira and North Africa. MADEIRA: locality unknown (China, 1938).

Habitat and food plants. Unknown.

\section{* Austroagallia sinuata (Mulsant \& Rey, 1855)}

Bythoscopus sinuatus Mulsant \& Rey, 1855: 222

Distribution. Widely distributed in Palaearctic including Canary Islands and Afrotropical region. MADEIRA: recorded from high altitudes on Pico Areeiro.

Habitat and food plants. Unknown.

MATERIAL EXAMINED: $10^{\star}$ Pousada do Pico do Arieiro, Fontal, Madeira, 1810 m, 21.iii.2001, leg. D. Aguin-Pombo.

\section{Subfamily Aphrodinae Blöte, 1927}

\section{Anoscopus assimilis (Signoret, 1879)}

Acocephalus assimilis Signoret, 1879: 75; Noualhier, 1897: 77; China, 1938: 44

Aphrodes assimilis; Lindberg, 1961: 67

Distribution. Europe and North Africa. BUGIO: locality unknown (China, 1938). DESERTA GRANDE: locality unknown (China, 1938). MADEIRA: locality unknown (China, 1938; Noualhier, 1897). PORTO SANTO: locality unknown (Lindberg, 1961).

Remarks. Information on this species is based on material sampled by Lindberg, Wollaston and Fauvel who did not report exact localities.

Habitat and food plants. Grasses.

\section{Anoscopus albifrons (Linnaeus, 1758)}

Cicada albifrons Linnaeus, 1758: 437

Acocephalus albifrons; China, 1938: 44

Aphrodes albifrons; Lindberg, 1961: 67

Distribution. Holoarctic region. MADEIRA: recorded from many localities from northern and southern slopes mainly above 800 m (China, 1938; Lindberg, 1961).

Habitat and/or food plants. Sampled on herbaceous plants and under roots (Lindberg, 1961). 


\section{? Aphrodes bicinctus (Schrank, 1776)}

Cicada bicincta Schrank, 1776: 75

Acocephalus nervosus; China, 1938: 44

Acocephalus striatus; Noualhier, 1897: 77

Aphrodes bicinctus; Lindberg, 1961: 67

Distribution. Europe and Madeira. MADEIRA: recorded from five localities on northern and southern slopes (China, 1938; Lindberg, 1961; Noualhier, 1897).

Habitat and food plants. Unknown.

Remarks. Several species have been recognized within the Aphrodes bicinctus complex (Le Quesne, 1988) but the interpretation followed by China, Lindberg or Noualhier is unknown, therefore, this record should be further confirmed.

Aphrodes brachypterus (China, 1938)

Acocephalus brachypterus China, 1938: 45

Distribution. Endemic to Madeira. MADEIRA: locality unknown (China, 1938).

Remarks. Described from a single female specimen in Wollaston's collection.

Habitat and food plants. Unknown.

\section{Subfamily Cicadellinae Latreille, 1825}

*Cicadella viridis (Linnaeus, 1758)

Cicada viridis Linnaeus, 1758: 438

Distribution. Holarctic and Oriental regions but not previously recorded from Macaronesia. MADEIRA:

Recorded from eastern part of Island at $650 \mathrm{~m}$.

Habitat and food plants. Unknown.

MATERIAL EXAMINED: 2 $q$ Couvinhas, Santo da Serra at 650m, Madeira, 14.xii.2001 leg., Fábio Reis.

\section{Subfamily Deltocephalinae Dallas, 1870}

Aconurella prolixa (Lethierry, 1885)

Thamnotettix prolixa Lethierry, 1885: 102

Aconurella prolixa; Lindberg, 1961: 64

Distribution. Palaearctic and Afrotropical regions. MADEIRA: reported from northern and southern slopes at different altitudes (Lindberg, 1961). PORTO SANTO: from southern seashore up to Serra de Dentro mountain (Lindberg, 1961).

Habitat. Moist or dry grass vegetation, often occurs in large numbers (Lindberg, 1961).

\section{Balclutha frontalis (Ferrari, 1882)}

Gnathodus frontalis Ferrari, 1882: 117

Balclutha rosea; Lindberg, 1961: 64

Distribution. Worldwide except Australian region. MADEIRA: sampled on seashore localities of southern slope (Lindberg, 1961).

Habitat. Found in dry areas on grasses (Lindberg, 1961).

*Balclutha hebe (Kirkaldy, 1906)

Nesosteles hebe Kirkaldy, 1906: 343

Distribution. Cosmopolitan. MADEIRA: recorded only from west coastal.

Habitat. Low grass vegetation. 
MATERIAL EXAMINED: $2 \sigma^{\pi} \sigma^{\pi}$, 1, Paul do Mar, Madeira, 14.viii.2005 on Poaceae, leg. E. Nunes.

\section{Balclutha pellucens Horváth, 1909}

Balclutha pellucens Horváth, 1909: 296; China, 1938: 47; Lindberg, 1961: 64

Dicraneura viridella Lindberg, 1941: 23

Distribution. Endemic to Macaronesian archipelagos except Cape Verde. MADEIRA: common all over the island (China, 1938; Lindberg, 1941; Lindberg, 1961).

Habitat. Low grass vegetation (Lindberg, 1961).

\section{* Balclutha rufofasciata (Merino, 1936)}

Agellus rufofasciatus Merino, 1936a: 381

Distribution. North Africa, Neotropical and Oriental regions. MADEIRA: recorded from Funchal on southern coast.

Habitat. Low grass vegetation.

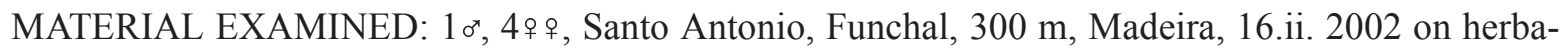
ceous plants, leg. Fábio Reis.

\section{Brachypterona vieirai Quartau, 1981}

Brachypterona vieirai Quartau, 1981: 127

Distribution. Endemic to Selvagens archipelago but not listed in recent checklists (Hoch, 2004). SEL-

VAGEM GRANDE: Pico da Atalaia (Quartau, 1981). SELVAGEM PEQUENA: reported from various localities (Quartau, 1981).

Habitat and/or food plants. On Lycopersicon esculentum in Selvagen Grande (Quartau, 1981) and in Selvagen Pequena on Agropyron junceiforme but present also in areas with Limonium papillatum, Suaeda vera, Frankenia laevis and Lotus salvagensis (Quartau, 1981).

\section{Cicadulina bipunctata (Melichar, 1904)}

Gnathodus bipunctata Melichar, 1904: 47

Cicadulina zeae; Lindberg, 1961: 63

Distribution. Worldwide except America. MADEIRA: recorded only from several localities of southern coast (Lindberg 1961).

Habitat and food plants. Unknown.

Remarks. An important pathogen vector in India, Africa and Philippines (Webb, 1987)

\section{Circulifer haematoceps (Mulsant \& Rey, 1855)}

Jassus haematoceps Mulsant \& Rey, 1855: 229

Circulifer haematoceps var. vittiventris; Lindberg, 1961: 65

Distribution. Palaearctic region including Macaronesia but here known only from Madeira. PORTO SANTO: locality unknown (Lindberg, 1961).

Habitat and food plants. Unknown.

Remarks. Circulifer haematoceps and C. opacipennis have been variously regarded as different species or the second as a synonym of the first. Here the interpretation of Young \& Frazier (1954) is followed and both species are considered valid. Circulifer haematoceps is an important vector in Mediterranean Basin and Near East (Kersting and Baspinar, 1995).

\section{Circulifer opacipennis (Lethierry, 1786)}

Cicadula opacipennis Lethierry, 1786: 12

Circulifer opacipennis; Quartau, 1984: 4

Distribution. Europe, North Africa and Near East. SELVAGEM PEQUENA: reported from several 
localities (Quartau, 1984).

Habitat and/or food plants. Areas with mixed vegetation including Agropyron junceiforme, Suaeda vera and Limonium papillatum (Quartau, 1984).

Remarks. An important vector of Spiroplasma citri (Kersting et al., 1992). See Circulifer haematoceps for other comments.

\section{Euscelidius variegatus (Kirschbaum, 1858)}

Athysanus variegatus Kirschbaum, 1858a: 9

Euscelidius variegatus; Reis and Aguin-Pombo, 2003: 28

Distribution. Palaearctic. MADEIRA: reported from coastal areas up to $1175 \mathrm{~m}$ on both slopes of Island (Reis and Aguin-Pombo, 2003).

Food plants. Brachypodium sylvaticum, Brachiaria mutica (Gramineae), Trifolium repens (Leguminosae) and Apium nodiflorum (Umbelliferae) (Reis and Aguin-Pombo, 2003).

Remarks. An important vector of phytoplasmas and other microorganisms (Palermo et al., 2001).

\section{? Euscelis incisus (Kirschbaum, 1858)}

Athysanus incisus Kirschbaum, 1858: 10

Athysanus plebejus; China, 1938: 46

Euscelis plebejus?; Lindberg, 1961: 66

Distribution. Palaearctic region including Macaronesia but here known only from Madeira. DESERTA GRANDE: locality unknown (China, 1938). ISLET CHÃO: locality unknown (China, 1938). PORTO SANTO: reported from few localities (China, 1938; Lindberg, 1961).

Remarks. This species shows seasonal variation in the morphology of genital structures (Muller, 1983). Lindberg (1961) examined China's material of E. incisus from Porto Santo and Desertas and identified it tentatively as E. plebejus but considered that could belong also to E. lineolatus Brullé. However, part of this material could belong also to E. ormaderensis Remane 1968 (see Remane, 1968) or to a different species. Euscelis incisus is a vector of several plant diseases (Della Giustina, 1989) and its presence on Madeira should be confirmed.

Habitat and food plants. Unknown.

\section{Euscelis ormaderensis Remane, 1968}

Euscelis ormaderensis Remane, 1968: 7

Distribution. Endemic to Madeira archipelago. DESERTA GRANDE: locality unknown (Remane, 1968). ISLET CHÃO: locality unknown (Remane, 1968). PORTO SANTO: reported from few localities (Remane, 1968).

Food plants. Herbs of several plant families but often associated to Artemisia argentea (Remane, 1968).

Remarks. The specific status of the specimens from Porto Santo was confirmed after being studied the seasonal variation in genital structures and acoustic behaviour. Some additional morphologically very variable specimens of Euscelis were found also by Remane in Madeira at Prazeres but their specific identity was not determined (Remane, 1968).

\section{Exitianus capicola (Stål, 1855)}

Athysanus capicola Stål, 1855: 9; China, 1938: 46

Athysanus taeniaticeps; Lindberg, 1941: 31

Exitianus capicola; Lindberg, 1961: 65; Quartau, 1984: 6

Distribution. Palaearctic and Afrotropical. MADEIRA: reported from several localities on both slopes of Island mainly at low and medium altitudes (China, 1938; Lindberg, 1941; Lindberg, 1961; Quartau, 1984).

Habitat. Agricultural areas invaded by grasses (Lindberg, 1961). A vector of phytoplasmas and/or 
spiroplasmas (Boukhris-Bouhachem et al., 2007).

\section{Exitianus fasciolatus (Melichar, 1911)}

Athysanus fasciolatus Melichar, 1911: 107

Exitianus vulnerans; Lindberg, 1961: 65

Exitianus fasciolatus; Quartau, 1984: 5

Distribution. West Palaearctic and Afrotropical. PORTO SANTO: locality unknown (Lindberg, 1961). SELVAGEM PEQUENA: Eastern part (Quartau, 1984).

Food plants. Agropyron junceiforme (Quartau, 1984).

\section{Macrosteles ossiannilssoni Lindberg, 1954}

Macrosteles ossiannilssoni Lindberg, 1954: 237; Lindberg, 1961: 63

Macrosteles sexnotatus; Lindberg, 1941: 31 [identification error]

Cicadula fasciifrons; China, 1938: 46 [Identification error]

Distribution. Europe. MADEIRA: reported from many localities from the coast up to mountain slopes (Lindberg, 1941). PORTO SANTO: reported only from Serra de Dentro-Pico Juliana (Lindberg, 1961).

Habitat. Wet shores of streams with rich vegetation (Lindberg, 1961).

Remarks. Lindberg (1941) examined 15 specimens of Cicadula fasciifrons Stål from Wollaston's collection, studied also by China (1938, p.46), and misidentified them as Macrosteles sexnotatus Fall. 1806. Later, Lindberg (1961) stated that this material could belong either to M. ossianilssoni, to $M$. fascifrons or to $M$. ramosus (= quadricornis). Because $M$. ossiannilssoni was very common in Madeira, Lindberg considered this material as probably M. ossiannilssoni. M. fascifrons is known from Scandinavia and Siberia; therefore, its presence in Madeira is unlikely. This record should be further confirmed.

\section{Macrosteles ramosus Ribaut, 1952}

Macrosteles ramosus Ribaut, 1952: 51

Macrosteles quadricornis Lindberg, 1954: 235; Lindberg, 1961: 63

Distribution. Southern Europe and Eastern Palaearctic. MADEIRA: recorded from two seashore localities in north and southern parts of island (Lindberg, 1961). PORTO SANTO: recorded only from Vila Baleira (Lindberg, 1961).

Habitat and food plants. Unknown.

\section{* Macrosteles sexnotatus (Fallén, 1806)}

Cicada sexnotata Fallén, 1806: 34

Distribution. Palaearctic including Azores and Canary Islands. MADEIRA: Western part of Madeira. Habitat and food plants. Unknown.

Remarks. This species was erroneously reported to Madeira as M. ossiannilssoni by Lindberg in 1941 (see Lindberg, 1961 and remarks for M. ossiannilssonni above).

MATERIAL EXAMINED: 10, 2q̊, Caniçal, Ponta de São Lourenço, Madeira, 01.i.1998, leg. D. Aguin-Pombo.

\section{? Mocuellus collinus (Boheman, 1850)}

Deltocephalus collinus Boheman, 1850a: 261; China, 1938: 46 [?]

Mocuellus collinus?; Lindberg 1961: 67

Distribution. Palaearctic except North Africa. MADEIRA: locality unknown (China, 1938 as Deltocephalus collinus Boh.?).

Remarks. This record is based on a single female from Wollaston's collection examined by China 
(1938) who stated that this specimen superficially resembled M. collinus. Later, Lindberg (1961) identified these specimens tentatively as Mocuellus collinus Boh. This record needs further confirmation.

Habitat and food plants. Unknown.

\section{Nesoclutha erythrocephala (Ferrari, 1882)}

Cicadula erythrocephala Ferrari, 1882: 118

Irinula erythrocephala; Lindberg, 1961: 64

Distribution. Palaearctic except North Africa. MADEIRA: reported only from Funchal (Lindberg, 1961).

Remarks. This rare species may have been introduced (Lindberg, 1961).

Habitat and food plants. Unknown.

\section{Opsius lethierryi Wagner, 1942}

Opsius lethierryi Wagner, 1942: 121; Lindberg, 1961: 65

Distribution. Western Palaearctic region including Canary Islands and North Africa. MADEIRA: reported only from Funchal (Lindberg, 1961).

Food plants. Tamarix (Lindberg, 1961).

\section{Orosius orientalis (Matsumura, 1914)}

Eutettix orientalis Matsumura, 1914: 192

Nesophrosyne cellulosa; Lindberg, 1961: 65

Distribution. Occurs in Afrotropical, Oriental and Palaearctic regions including Canary Islands and Madeira but not listed in recent checklist (Hoch, 2004). MADEIRA: known from one specimen from Serra de Agua in southern part of island (Lindberg, 1961).

Remarks. This is one of the best known vectors of plant diseases caused by phytoplasmas and also vector of viruses diseases (Horn et al., 1994).

Habitat and food plants. Unknown.

\section{Psammotettix striatus (Linnaeus, 1758)}

Thamnotettix striatus Linnaeus, 1758: 437

Psammotettix alienus; Lindberg, 1961: 66

Deltocephalus striatus; China, 1938: 46 [identification error]

Psammotettix striatus; Lindberg, 1941: 31 [identification error]

Distribution. Holarctic. DESERTA GRANDE: locality unknown (Lindberg, 1961). MADEIRA: Reported from many localities from coastal areas up to mountain tops on northern and southern slopes (Lindberg, 1961; China, 1938; Lindberg, 1941). PORTO SANTO: reported from three localities from coastal areas up to high altitudes in northern slopes (Lindberg, 1961).

Habitat and/or food plants. Herbaceous vegetation and in the outside of cultivated terrains (Lindberg, 1961).

Remarks. China (1938) identified the material of Wollaston's collection as Deltocephalus striatus (= Psammotettix striatus) and Lindberg (1941) identified it as Psammotettix striatus. Later, Lindberg (1961) reexamined this material and considered it to be P. alienus. Presently Psammotettix alienus is considered a synonym of Psammotettix striatus (Razvyazkina \& Pridantseva, 1968). This species is a vector of viruses and phytoplasms which cause plant diseases (Della Giustina, 1989).

\section{*Recilia angustisectus (Linnavuori, 1962)}

Deltocephalus angustisectus Linnavuori, 1962: 55

Distribution. Previously known only from Israel (Linnavuori, 1962). MADEIRA: reported from eastern and driest part of Madeira (Ponta de São Lourenço). 
Habitat. Herbaceous vegetation.

MATERIAL EXAMINED: 2 ơ $^{\star}$, Ponta de São Lourenço, Madeira, 1.iv.2001, leg. D. Aguin-Pombo.

\section{Subfamily Eupelicinae Shalberg, 1871}

\section{Chloropelix canariensis Lindberg, 1936}

Chloropelix canariensis Lindberg, 1936: 4; Lindberg, 1961: 67

Distribution. Macaronesia, North Africa and Israel not listed for Madeira in recent checklists (Nast, 1972; Hoch, 2007). MADEIRA: reported only from Funchal (Lindberg, 1961).

Habitat and food plants. Unknown.

\section{Subfamily Typhlocybinae Kirschbaum, 1868}

\section{Alebra viridis Rey, 1897}

Alebra albostriella var. viridis Rey, 1897: 46

Alebra viridis; Aguin-Pombo, 2002: 68

Distribution. A European species not listed for Madeira archipelago in recent checklists (Hoch, 2004). MADEIRA: found in naturalized chestnut forests above $300 \mathrm{~m}$ altitude on southern slopes (Aguin-Pombo, 2002).

Food plants. Quercus robur and Castanea sativa (Aguin-Pombo, 2002).

Remarks. Some females recorded by Lindberg from Madeira as Alebra albostriella (Fallén, 1826) were deposited at the Municipal Museum of Funchal, but these resemble A. viridis (Aguin-Pombo, 2002). This last species was considered a variety of $A$. albostriella at the time that Lindberg published his work. The occurrence of $A$. albostriella on Madeira is unlikely.

\section{Asianidia albula (Lindberg, 1961)}

Erythroneura albula Lindberg, 1961: 73

Distribution. Endemic to Madeira. MADEIRA: reported from one locality on southern slope (Lindberg, 1961).

Habitat and food plants. Unknown.

\section{Asianidia atlantica (China, 1938)}

Erythroneura atlantica China, 1938: 50; China, 1938: 50; Lindberg, 1961: 73

Asianidia atlantica; Quartau and Remane, 1996: 2

Distribution. Endemic to Madeira archipelago. ISLET CHÃO: locality unknown (China, 1938). MADEIRA: reported from coastal areas up to medium altitude on southern slopes (Lindberg, 1961). PORTO SANTO: Serra de Dentro-Juliana (Lindberg, 1961)

Food plants. Artemisia argentea and Artemisia arborescens (Lindberg, 1961; Quartau and Remane, 1996).

\section{Asianidia chinai (Lindberg, 1961)}

Erythroneura chinai Lindberg, 1961: 72.

Asianidia chinai; Quartau et al., 2004: 161

Distribution. Endemic to Madeira archipelago. MADEIRA: reported from two localities at high altitudes (Lindberg, 1961; Quartau et al., 2004).

Habitat and/or food plants. Isoplexis sceptrum, an endemic species of the native laurel forest (Quartau et al., 2004). 


\section{Asianidia chrysanthemi (Lindberg, 1954)}

Erythroneura chinai Lindberg, 1954: 252; Lindberg, 1961: 252

Asianidia chrysanthemi; Quartau and Remane, 1996: 2

Distribution. Endemic to Madeira archipelago and Canary Islands. MADEIRA: reported from five localities of southern slope (Lindberg, 1961, Quartau and Remane, 1996). PORTO SANTO: locality unknown (Lindberg, 1961).

Food plants. Argyranthemum pinnatifidium, an endemic species (reported by Lindberg, 1961 and Quartau and Remane, 1996 as Chrysanthemum pinnatifidium).

\section{Asianidia decolor (Lindberg, 1936)}

Erythroneura decolor Lindberg, 1936: 15; China, 1938: 49; Lindberg, 1961: 72

Distribution. Endemic to Madeira archipelago and Canary Islands. MADEIRA: reported from localities above 300m on southern slopes (China, 1938; Lindberg, 1961).

Food plants. Labiatae spp. (Lindberg, 1961).

\section{Asianidia insulana (Lindberg, 1961)}

Erythroneura insulana Lindberg, 1961: 71

Distribution. Endemic to Madeira Island. MADEIRA: on seashore on southern slopes (Lindberg, 1961).

Habitat. Native laurel forest (Lindberg, 1961).

\section{Asianidia madeirensis (China, 1938)}

Erythroneura madeirensis China, 1938: 48; Lindberg, 1941: 31; Lindberg, 1961: 69

Distribution. Endemic to Madeira archipelago. MADEIRA: reported from many localities all over island, mainly above 500m (China, 1938; Lindberg, 1941; Lindberg, 1961).

Habitat. Shady places in forests (Lindberg, 1961).

\section{Asianidia melliferae Quartau \& Remane, 1996}

Asianidia melliferae Quartau \& Remane, 1996: 2

Distribution. Endemic to Madeira Island but not included in recent checklists (Hoch, 2004).

MADEIRA: known from a single locality (Ribeiro Bonito) on northern slope (Quartau and Remane, 1996).

Food plants. Probably monophagous on Euphorbia mellifera, a rare endemic plant of Macaronesian laurel forest growing in moist shady places (Quartau and Remane, 1996).

\section{Asianidia vallicola (Lindberg, 1954)}

Erythroneura vallicola Lindberg, 1954: 252; Lindberg, 1961: 73

Distribution. Endemic to Canary Islands and Madeira archipelago. MADEIRA: Islet Fora: a small islet located at eastern part of Madeira Island (Lindberg, 1961).

Habitat. Halophyte plants (Lindberg, 1961).

\section{Asymmetrasca decedens (Paoli, 1932)}

Empoasca decedens Paoli, 1932: 117

Asymmetrasca decedens; Freitas and Aguin-Pombo, 2004: 103

Distribution. Palaearctic and Oriental regions (Freitas and Aguin-Pombo, 2005), not listed for Madeira in recent checklists (Hoch, 2004). MADEIRA: all over island (Freitas and Aguin-Pombo, 2004).

Food plants. Exotic ornamental plants such as Ricinus communis, Lavatera cretica, Passiflora edulis, Tagetes minuta, Schinus molle, Enterolobium cyclocarpium and Dahlia sp. (Freitas and AguinPombo, 2004). 
Remarks. Probably recently introduced to Madeira Island (Freitas and Aguin-Pombo, 2004).

\section{Empoasca alsiosa Ribaut, 1936}

Empoasca alsiosa Ribaut, 1936: 155.

Empoasca unicolor; Lindberg, 1961: 75

Distribution. Southern Europe and Macaronesia. MADEIRA: known from central parts (Lindberg, 1961).

Habitat. Native forest (Lindberg, 1961).

\section{Empoasca fabalis DeLong, 1930}

Empoasca fabalis DeLong, 1930: 92; Aguin-Pombo et al., 2006: 172

Distribution. An American species not recorded from Europe (Nast, 1972; Hoch, 2007). MADEIRA: locality unknown (Aguin Pombo et al., 2006).

Remarks. Chromosomes of E. fabalis were studied in material from Madeira but this record has not been published (Aguin Pombo et al., 2006).

Habitat and food plants. Unknown.

\section{Empoasca distinguenda Paoli, 1932}

Empoasca distinguenda Paoli, 1932a: 114

Distribution. An African species not recorded from Europe (Nast, 1972; Hoch, 2007). MADEIRA: locality unknown (Aguin Pombo et al., 2006).

Remarks. Identified based on 5 males. Parthenogenetic females probably of E. distinguenda were studied cytogenetically but this record has not been published (Aguin Pombo et al., 2006).

Habitat and food plants. Unknown.

\section{? Eupteryx capreola Lindberg, 1954}

Eupteryx capreola Lindberg, 1954: 240; Quartau and Fançony, 1988: 98; Aveiro and Aguin-Pombo, 2003a, $2003 b$

Distribution. Endemic to Canary Islands and Madeira archipelago. MADEIRA: reported from many localities of southern and northern slopes (Lindberg, 1961; Quartau and Fançony, 1988). PORTO SANTO: one locality (Quartau and Fançony, 1988).

Food plants. Pteridium aquilinum and herbaceous plants (Quartau and Fançony, 1988).

Remarks. Quartau and Fançony (1988) suggested that Eupteryx capreola Lindberg, 1954 is probably an extreme form of Eupteryx filicum (Newman, 1853). More recent morphological studies and population dynamics analysis of populations on $P$. aquilinum also failed to separate this species from $E$. filicum (Aveiro and Aguin-Pombo, 2003a, 2003b). The status of this species needs to be confirmed.

\section{Eupteryx filicum (Newman, 1853)}

Typhlocyba filicum Newman, 1853: 132

Eupteryx laureti; China, 1938: 47; Lindberg, 1941: 31

Eupteryx filicum; Lindberg, 1961: 74

Distribution. Europe and Macaronesia. MADEIRA: reported from many localities at medium and high altitudes of northern and southern slopes (Lindberg, 1941; Lindberg, 1961). PORTO SANTO: sampled by Wollaston (China, 1938 as E. laureti Lind.) and Lindberg in Pico Castelo-Pico do Facho mountains (Lindberg, 1961).

Habitat. Native forest areas and above this forest on Pteridium aquilinum (Lindberg, 1961 as Eupteris aquilina).

Remarks. See remarks for E. capreola Lindberg, 1954. 
*Tamaricella cf. fasciolata (Lethierry, 1876)

Typhlocyba fasciolata Lethierry, 1876: 449

Distribution. Palaearctic, including Canary Islands and North Africa, and Afrotropical regions. MADEIRA: reported from southern coast on western part of island.

Food plants. Tamarix sp.

MATERIAL EXAMINED: $20^{\pi} 0^{\pi}, 4 ㅇ ㅜ$, Paul do Mar, Madeira, 14-viii-2005, on Tamarix sp, leg. E. Nunes.

Typhlocyba maderae Lindberg, 1961

Typhlocyba maderae Lindberg, 1961: 74

Distribution. Endemic to Madeira Island. MADEIRA: at high altitudes in native forest (Lindberg, 1961).

Food plants. Erica spp. (Lindberg, 1961).

*Zyginidia lineata (Lindberg, 1954)

Erythroneura lineata Lindberg, 1954: 245

Distribution. A Mediterranean species present in Canary Islands (Lindberg, 1954, Nast, 1972).

MADEIRA: reported from laurel forest at high altitudes.

Food plants. Grasses.

MATERIAL EXAMINED: $10^{\star}, 1$ \% , Ribeiro Frio-Poiso, Madeira, 1000m, 05.ix.1996, on Poaceae, leg. D. Aguin-Pombo.

\section{Subfamily Evacanthinae Metcalf, 1939}

\section{Sophonia orientalis (Matsumura, 1912)}

Nirvana orientalis Matsumura, 1912: 282

Sophonia orientalis; Li and Chen, 1999: 51; Aguin-Pombo et al., 2006: 21-22

Distribution. Oriental species recently introduced to Madeira archipelago and Canary Islands where is becoming an invasive species (Aguin-Pombo et al., 2006; Webb and Viraktamath. 2004). MADEIRA: reported from many localities of southern part (Aguin-Pombo et al., 2006).

Food plants. A polyphagous species feeding on many plant species of Anacardiaceae, Passifloraceae, Asteraceae, Euphorbiaceae, Lauraceae, Myrtaceae, Malvaceae, Convolvulaceae, Geraniaceae, Zingiberaceae, Polygonaceae, Solanaceae, Fabaceae, Globulariaceae and Caricaceae (Aguin-Pombo et al., 2006).

\section{Subfamily Penthimiinae Kirschbaum, 1868}

\section{Penthimia irrorata Horváth, 1909}

Penthimia irrorata Horváth, 1909: 297; Lindberg, 1961: 68

Distribution. Endemic to Canary Islands and Madeira archipelago but not listed in recent checklists (Hoch, 2004). MADEIRA: reported only from Serra de Água (Lindberg, 1961).

Habitat and food plants. Unknown. 


\title{
Suborder Fulgoromorpha Evans, 1946
}

\author{
Family Cixiidae Spinola, 1839
}

\section{Subfamily Cixiinae Spinola, 1839}

\section{Cixius chaoensis China, 1938}

Cixius chaoensis China, 1938: 52; Lindberg, 1961: 55

Distribution. Endemic to Madeira archipelago. ISLET CHÃO: locality unknown (China, 1938; Lindberg, 1961).

Habitat and food plants. Unknown.

\section{Cixius madeirensis China, 1938}

Cixius madeirensis China, 1938: 51; Lindberg, 1961: 55

Distribution. Endemic to Madeira archipelago. MADEIRA: reported from several mountain localities on northern and southern slopes (China, 1938; Lindberg, 1961).

Habitat. Native vegetation (Lindberg, 1961).

\section{Cixius verticalis Noualhier, 1897}

Cixius verticalis Noualhier, 1897: 79; China, 1938: 50; Lindberg, 1941: 31; Lindberg, 1961: 55

Distribution. Endemic to Madeira archipelago. MADEIRA: occurs at medium and high altitudes mainly on northern slopes (China, 1938; Lindberg, 1941; Lindberg, 1961; Noualhier, 1897).

Habitat. Hedges and trees in native forest (Lindberg, 1961).

\section{Hyalesthes madeires Remane \& Hoch, 1986}

Hyalesthes madeires Remane \& Hoch, 1986: 132; Hoch and Remane, 1985: 132

Hyalesthes flavipennis; Lindberg, 1961: 54-55 [identification error]

Distribution. Endemic to Madeira Island. MADEIRA: reported from several localities on northern and southern slopes up to $600 \mathrm{~m}$ (Hoch and Remane, 1985; Lindberg, 1961; Remane and Hoch, 1986).

Habitat and/or food plants. Globularia spp., Echium spp., Rubus spp., Euphorbia spp. and other xerophyte vegetation (Hoch and Remane, 1985; Remane and Hoch, 1986).

Remarks. Lindberg (1961) identified 83 specimens from Serra de Agua as Hyalesthes flavipennis but Hoch \& Remane (1985) studied part of this material (74 specimens) and identified them as a new species, H. madeires.

\section{Hyalesthes portonoves Remane \& Hoch, 1986}

Hyalesthes portonoves Remane \& Hoch, 1986: 134; Hoch and Remane, 1985: 134

Distribution. Endemic to Madeira Island. MADEIRA: reported from a single southern coastal locality (Porto Novo) (Hoch and Remane, 1985; Remane and Hoch, 1986).

Habitat and/or food plants. Xerophyte vegetation with Echium spp. and Euphorbia spp. (Hoch and Remane, 1985; Remane and Hoch, 1986).

Remarks. Lindberg (1941) identified two specimens from Portonovo as Hyalesthes angustulus Horv. 1908 but later (1961) he identified them as Hyalesthes flavipennis. Remane and Hoch (1985) described $H$. portonoves from the same locality; therefore, it is very likely that Lindberg's material corresponds to this species. 


\section{Family Delphacidae Leach, 1815}

\section{Subfamily Delphacinae Leach, 1815}

Flastena fumipennis (Fieber, 1866)

Delphax fumipennis Fieber, 1866: 527

Calligypona bifurcata; Lindberg, 1961: 62.

Distribution. Palaearctic. MADEIRA: known only from a coastal locality (São Vicente) in northern part (Lindberg, 1961).

Habitat. Grasses and other vegetation growing on the margin of a stream (Lindberg, 1961).

Florodelphax leptosoma (Flor, 1861)

Delphax leptosoma Flor, 1861: 76

Calligypona leptosoma; Lindberg, 1961: 60

Distribution. Europe and Near East. MADEIRA: reported from two coastal localities on northern and southern parts (Lindberg, 1961).

Habitat and food plants. Unknown.

\section{Javesella dubia (Kirschbaum, 1868)}

Delphax dubia Kirschbaum, 1868: 26

Delphacodes pellucida; Lindberg, 1941: 32

Calligypona dubia; Lindberg, 1961: 60

Javesella dubia; Remane, 1975: 1

Distribution. Widely distributed in Palaearctic region except North Africa. MADEIRA: reported from coast to mountains in northern and southern slopes (Lindberg, 1941; Lindberg, 1961).

Remarks. The presence of this species in Madeira was further confirmed by Remane (1975).

Habitat and food plants. Unknown.

\section{Laodelphax striatellus (Fallén, 1826)}

Delphax striatellus Fallén, 1826: 129

Delphacodes striatella; China 1938: 54

Delphacodes striatellus; Lindberg, 1941: 32

Calligypona marginata; Lindberg, 1961: 60 [erroneous synonym]

Distribution. Palaearctic except North Africa, and Oriental and Afrotropical regions. MADEIRA: reported from several localities from different altitudes in northern and southern slopes (China 1938; Lindberg, 1941; Lindberg, 1961).

Remarks. China (1938) recorded this species as Delphacodes striatella Fall. Later, Lindberg (1961) identified his own material published in 1941 and China's material as Delphacodes striatella Fall.. but erroneously misplaced this species as a synonym of Calligypona marginata (Fabr.). This latter species is a synonym of Javesella pellucida Fabricius, 1794.

Habitat and food plants. Unknown.

Remarks. A vector of the rice stripe virus (Lin et al., 1996)

\section{Liburnia anthracina Horváth, 1909}

Liburnia anthracina Horváth, 1909: 301

Calligypona anthracina; Lindberg, 1961: 61

Distribution. An Afrotropical species present in Macaronesia. MADEIRA: known only from Funchal and Ribeira Brava, on southern and northern coast, respectively (Lindberg, 1961).

Habitat and food plants. Unknown. 


\section{Makarorysa madalta Remane \& Asche, 1986}

Makarorysa madalta Remane \& Asche, 1986: 228

Distribution. Endemic to Madeira. MADEIRA: reported from a single locality at $1450 \mathrm{~m}$ in eastern part of Pico do Areeiro Mountain (Remane and Asche, 1986).

Habitat and/or food plants. Meadowlike clearing feeding probably on grasses (Remane and Asche, 1986).

Remarks. The specimens identified by Lindberg as Eurysa ribauti (Lindberg, 1936) probably correspond to M. madalta and/or to M. madeco (see Remane and Asche, 1986).

Makarorysa madeco Remane \& Asche, 1986

Makarorysa madeco Remane \& Asche, 1986: 227

Distribution. Endemic to Madeira Island. MADEIRA: reported from coastal areas up to $1800 \mathrm{~m}$ (Pico Ruivo) (Remane and Asche, 1986).

Food plants. Grasses (Remane and Asche, 1986).

\section{Megamelodes quadrimaculatus (Signoret, 1865)}

Delphax quadrimaculatus Signoret, 1865: 5

Megamelus fieberi; Lindberg, 1961: 59

Distribution. Europe, North Africa and Macaronesia. MADEIRA: reported from coast up to high atitudes in northern and southern slopes (Lindberg, 1961).

Habitat. Shady and moist places of native forest areas (Lindberg, 1961).

\section{Muellerianella fairmairei (Perris, 1857)}

Delphax fairmairei Perris, 1857: 170

Calligypona fairmairei; Lindberg, 1961: 62

Distribution. Palaearctic including Azores and Madeira archipelagoes. MADEIRA: common at various altitudes on northern and southern slopes (Lindberg, 1961).

Habitat and food plants. Unknown.

\section{Pseudaraeopus lethierryi (Mulsant \& Rey, 1879)}

Araeopus lethierryi Mulsant \& Rey, 1879: 319

Pseudaraeopus lethierryi; Lindberg, 1961: 59

Distribution. Widely distributed in Mediterranean Basin, Near East and Macaronesia. MADEIRA: known only from Funchal (Lindberg, 1961).

Habitat and food plants. Unknown.

\section{Sogatella nigeriensis (Muir, 1920)}

Megamelus nigeriensis Muir, 1920: 140; Asche and Wilson, 1990: 13

Distribution. Occurs in Palaearctic and Afrotropical regions. MADEIRA: locality unknown (Asche and Wilson, 1990). The material of Sogatella vibix and S. furcifera studied by Lindberg (1961) and deposited in the British Museum, was identified by Asche and Wilson (1990) as S. nigeriensis.

Remarks. All material of Sogatella furcifera (Horváth, 1889) from Madeira reported by China (1938) and Lindberg (1961) and that of Sogatella vibix (Haupt, 1927) reported by Lindberg (1961) could correspond either to S. nigeriensis or S. kolophon Kirkaldy, 1906 (see Asche and Wilson, 1990). The presence of $S$. kolophon should be further confirmed.

Habitat and food plants. Unknown.

\section{Toya hispidula (Lindberg, 1954)}

Calligypona hispidula Lindberg, 1954: 187; Lindberg, 1961: 61

Toya hispidula; Asche, 1980: 8 
Distribution. African and Macaronesia. MADEIRA: known from a single locality, Porto da Cruz, in northern coast (Lindberg, 1961). PORTO SANTO: locality unknown (Asche, 1980).

Habitat. Culture zone (Lindberg, 1961).

\section{Toya propinqua (Fieber, 1866)}

Delphacodes propinqua Fieber, 1866: 525; China, 1938: 54

Calligypona propinqua; Lindberg, 1961: 60

Delphacodes albicollis; Lindberg, 1941: 32 [identification error]

Distribution. Worldwide except Australian region. MADEIRA: reported from many localities at different altitudes (China, 1938, no localities indicated; Lindberg, 1941; Lindberg, 1961). PORTO SANTO: sampled from southern coast up to high altitudes (Lindberg, 1961).

Habitat. In grass places and cultivated areas (Lindberg, 1961).

Remarks. This species was misidentified by Lindberg (1941) as Delphacodes albicollis (Motschulsky, 1863) but subsequently corrected by him (Lindberg, 1961).

\section{Subfamily Kelisiinae Wagner, 1963}

\section{Kelisia ribauti Wagner, 1938}

Kelisia ribauti Wagner, 1938: 12; Lindberg, 1941: 25; Lindberg, 1961: 59

Distribution. Palaearctic region including Macaronesia. MADEIRA: sampled in two southern localities (Lindberg, 1941; Lindberg, 1961).

Habitat and food plants. Unknown.

\section{Family Dictyopharidae Spinola, 1839}

\section{Subfamily Orgeriinae Fieber, 1872}

\section{Almana longipes (Dufour, 1849)}

Dictyophara longipes Dufour, 1849: 98

Almana longipes; Schmidt, 1915: 358

Distribution. Iberian Peninsula, North Africa and Madeira. MADEIRA: locality unknown (Schmidt, 1915).

Habitat and food plants. Unknown.

\section{Family Flatidae Spinola, 1839}

\section{Subfamily Flatinae Melichar, 1903}

\section{Cyphopterum fauveli (Noualhier, 1897)}

Hysteropterum fauveli Noualhier, 1897: 79.

Cyphopterum fauveli; Melichar, 1905: 476; China, 1938: 53; Lindberg, 1961: 58; Leise, 1992: 259

Distribution. Endemic to Madeira archipelago. DESERTA GRANDE: locality unknown (Leise, 1992; Lindberg, 1961). MADEIRA: sampled on southern coast in Funchal and Ponta de São Lourenço, the driest part of the Island (Noualhier, 1897; Melichar, 1905; Lindberg, 1961). Islet de Fora: locality unknown (Lindberg, 1961). ISLET CHÃO: locality unknown (China, 1938; Leise, 1992; Lindberg, 1961). PORTO SANTO: Serra de Dentro and Pico Juliana (Lindberg, 1961; Leise, 1992). Ilhéu da 
Cevada: locality unknown (Lindberg, 1961).

Food plants. On halophyte plants (Lindberg, 1961).

Remarks. Leise (1992) indicates that Horváth's (1909) records of this species from La Palma (Canary Islands) correspond to C. grossum Lindberg, 1954 and Lallemand (1929) and Seabra's (1930a, 1930b, 1939a, 1939b, 1941) records from Portugal of the same species correspond to C. adscendens HerrichSchäeffer, 1835.

\section{Cyphopterum quartaui Linnavuori, 1974}

Cyphopterum quartaui Linnavuori, 1974: 2; Quartau, 1975: 2; Leise, 1992: 269

Distribution. Endemic to Salvagens archipelago. SELVAGEM PEQUENA: Pico do Veado (Linnavuori, 1974; Quartau, 1975; Leise, 1992)

Food plants. Suaeda vera and Agropyron junceiforme (Quartau, 1975).

\section{Cyphopterum retusum (Walker, 1851)}

Issus retusus Walker, 1851: 371

Cyphopterum retusum; Melichar, 1905: 477; China, 1938: 54; Leise, 1992: 260; Lindberg, 1961: 57

Cyphopterum obtusatum; Leise, 1992: 9

Distribution. Endemic to Madeira archipelago. MADEIRA: reported from several localities in northern and southern slopes at different altitudes (China, 1938; Leise, 1992; Lindberg, 1961; Walker, 1851 as Issus retusus; Melichar, 1905).

Habitat and/or host plant. Apparently polyphagous and common in hedges, in many cases on Rubus (Lindberg, 1961) but present also in many types of habitats and vegetation including pine forest with Faya, Acacia, coastal vegetation Rubus and Artemisia, herbaceous vegetation with Pterys and Rubus, moist hillsides with Salix, Ulex and Rubus (Leise, 1992).

Remarks. This species was misidentified by several authors and erroneously reported from several localities. From mainland Portugal (Leiria) it was erroneously reported by Lallemand (1929a and 1929b) and Seabra (1930a, 1930b, 1939a, 1939b, 1941) based on an incorrect identification of $C$. adscendens (H.-S.) (see Leise, 1992). Another erroneous record seems to be "Port Philip", the type locality of $C$. curvipenne Walker 1958. Distant (1910) considered this species a synonym of $C$. retusum although he apparently did not examine the type material. The locality Port Philip is unknown to Madeira but known to Australia (Victoria) where this genus is absent. The Australian locality may have been wrongly assigned by Captain Parry, the donor of the specimen, who may have travelled from Madeira to Australia. This species seems also to have been misidentified as C. obtusatum Melichar, 1923; the description of this last species was based on a single female specimen from Cape Verde (Melichar, 1923), the only material of this species known. Leise (1922) after examining C. obtusatum could not find any differences in head or body shape from C. retusum (Leise, 1992). For Cape Verde seven endemic species of Cyphopterum were reported and all except $C$. obtusatum belong to a different subgenus, Phocypterum. Therefore, Leise (1992) suggested that this specimen was mislabelled and that this was the same species recorded from Madeira because it seems unlikely that a species of Cyphopterum s. str. occurs in Cape Verde. Additional support for this is the fact that this species has never been collected in further sampling by Lindberg who collected intensively on this archipelago.

\section{Cyphopterum salvagensis Lindberg, 1959}

Cyphopterum salvagensis Lindberg, 1959: 20; Leise 1992: 263; Quartau 1975: 2

Distribution. Endemic to Salvage archipelago. SELVAGEM PEQUENA: Pico do Veado (Lindberg, 1959; Quartau, 1975; Leise, 1992).

Food plants. Suaeda vera and Agropyron junceiforme (Leise 1992; Quartau 1975). 


\title{
Family Issidae Spinola, 1839
}

\section{Subfamily Issinae Spinola, 1839}

\section{Issus maderensis Lindberg, 1956}

Issus maderensis Lindberg, 1956: 58; Lindberg, 1961: 55; Remane, 1985: 94, 116, 120, 128, 130

Issus canariensis; China, 1938: 53 [identification error]; Lindberg, 1941: 32 [identification error].

Distribution. Endemic to Madeira Island. MADEIRA: reported from both slopes mainly at medium and high altitudes (China, 1938; Lindberg, 1941, 1956, 1961; Remane, 1985).

Food plants. Mainly on trees being very common on Erica (Lindberg, 1961).

Remarks. All material identified as I. canariensis by China (1938) corresponded to I. maderensis (Lindberg 1956, 1961).

\section{Doubtful records}

CICADELLIDAE Aphrodes bicinctus (Schrank, 1776)

Mocuellus collinus (Boheman, 1850)

Euscelis incisus (Kirschbaum, 1858)

Eupteryx capreola Lindberg, 1954

\section{Excluded species}

\author{
CICADELLIDAE Alebra albostriella (Fallén, 1826) \\ Macrosteles fasciifrons (Stål, 1858) \\ DELPHACIDAE Sogatella furcifera (Horváth, 1889) \\ Makarorysa ribauti (Lindberg, 1936) \\ Sogatella vibix (Haupt, 1927) \\ Javesella pellucida (Fabricius 1794)
}

\section{ACKNOWLEGMENTS}

We wish to thank many students that provided samples especially Élvio Nunes, Fábio Reis and Nelio Freitas. In addition we wish to thank A. F. Emeljanov for helping with identification of Recilia, Manfred Asche, for their comments on several species and to C. Dietrich for the improvement of the mansucript. We are thankful also to many colleagues who provide us literature and to T. Dellinguer for the translation of several German texts. This study was financed partially by a Synthesys grant to visit the Museum and Institute of Zoology of the Polish Academy of Sciences.

\section{REFERENCES}

Aguin-Pombo, D. (2002) Genetic differentiation among host associated Alebra leafhoppers (Hemiptera: Cicadellidae). Heredity, 88, 415-422. 
Aguin-Pombo, D. (2002) Validation of the faunistic data on the genus Alebra Fieber, 1872 (Hemiptera, Cicadellidae) in the Iberian Peninsula and Madeira. Boletín de la Sociedad Entomológica Aragonesa, 31, 67-70.

Aguin-Pombo, D., Oromi, P. \& Martin, E. (2005) Hemiptera: Auchenorrhyncha. In: Arechavaleta, M., N. Zurita, M.C. Marrero \& J.L. Martín (Eds.). Lista preliminar de especies silvestres de Cabo Verde (hongos, plantas y animales terrestres). 2005. Consejería de Medio Ambiente y Ordenación Territorial, Gobierno de Canarias, pp. 72-74.

Aguin-Pombo, D., Kuznetsova, V. \& Freitas, N. (2006) Multiple parthenoforms of Empoasca leafhoppers from Madeira Island: Where are these unisexual forms coming from? Journal of Heredity, 97 (2), 171-176.

Aguin-Pombo, D., Franquinho Aguiar, A. \& Kuznetsova, V. (2007) Bionomics and taxonomy of leafhopper Sophonia orientalis (Hemiptera: Cicadellidae), a Pacific pest species in the Macaronesian archipelagos. Annals of the Entomological Society of America, 99 (6), 19-26.

Asche, M. (1980) Zwei neue Arten der Gattung Toya Distant, 1906, aus dem Mittelmeergebiet mit erganzenden Bemerkungen zu Toya hispidula (Lindberg, 1953) und Toya obstusangula (Linnavuori, 1957) (Homoptera, Cicadina, Delphacidae). Marburger Entomologische Publikationen, 1(4), 1-36.

Asche, M. \& Wilson, M.R. (1990) The Delphacid genus Sogatella and related groups: a revision with special reference to rice-associated species (Homoptera: Fulgoroidea). Systematic Entomology, 15, 1-42.

Aveiro, M.J. \& Aguin-Pombo, D. (2003a) Population dynamics of Eupteryx leafhoppers on Pteridium aquilinum ferns in Madeira. How many species do we have? II Symposium of Island Ecosystems, Funchal, Madeira 5-9th October 2003, 71 .

Aveiro, M.J. \& Aguin-Pombo, D. (2003b) Large morphological variation in island populations of Eupteryx leafhoppers from Madeira Island. II Symposium of Island Ecosystems, Funchal, Madeira, 5-9th October 2003, 54.

Boukhris-Bouhachem, S., Chabbouh, N., Mounira, Harbi \& Jean-Luc, Danet (2007). The leafhoppers potential vectors of phytopathogene agents in Tunesian vineyards (Hemiptera: Cicadomorpha: Fulguromorpha). Annales de la Société Entomologique de France, 43(2), 159-163.

China, W.E. (1938) Die Arthropodenfauna von Madeira nach den Ergebnissen der reise von Prof. Dr. O. Lundblad. JuliAugust 1935. III. Terrestrial Hemiptera (Hemiptera and Homoptera Auchenorrhyncha). Arkiv för Zoologi, 30A (2), $1-69$.

Della Giustina W. (1989) Homoptères Cicadellidae, vol.3 compléments, INRA, Paris, 350 pp.

Distant, W.L. (1910) Rhynchotal notes. The Annals and Magazine of Natural History, 5 (8), 207-323.

Frankhan, R. (1997) Do island populations have less genetic variation than mainland populations? Heredity, 78, 311327.

Freitas, N. \&. Aguin-Pombo, D. (2004) Is the leafhopper Asymmetrasca decedens (Paoli, 1932) invading Madeira Island? Annales de la Société Entomologique de France, 40 (1), 103-104.

Freitas, N. \& Aguin-Pombo. D. (2005) Distribution, food plants and control of Asymmetrasca decedens (Paoli, 1932) (Hemiptera: Cicadellidae). Boletim do Museu Municipal do Funchal, 56 (315), 23-39.

Hoch, H. (Ed.) (2004) Hemiptera: Fulgoromorpha, Cicadomorpha. Fauna Europaea version 1.3, available from http:// www.faunaeur.org. (03.July.2007).

Hoch, H. \& Remane, R. (1985) Evolution und speziation der zikaden - gattung Hyalesthes Signoret, 1865 (Homoptera, Auchenorrhycha, Fulgoroidea, Cixiidae). Marburger Entomologische Publikationen, 2 (2), 1-427.

Horn, N.M., Reddy, S.V. \& Reddy, D.V.R. (1994) Virus-vector relationships of chickpea chlorotic dwarf geminivirus and the leafhopper Orosius orientalis (Hemiptera: Cicadellidae). Annals of Applied Biology, 124 (3), 441-450.

Horváth, G. (1909) Hémiptères recueillis par M. Th. Becker aux Iles Canaries. Annales Musei Nationalis Hungarici, 7 , 289-301.

Kersting, U. \& Baspinar, H. (1995) Seasonal and diurnal flight activity of Circulifer haematoceps (Hom., Cicadellidae), an important leafhopper vector in the Mediterranean area and the Near East. Journal of Applied Entomology, 119(8), 533-537.

Kersting, U., Sengonca, C. \& Cinar, A. (1992) Detection of Spiroplasma citri in non-citrus host plants and their associated leafhopper vectors in southern Turkey. FAO (Food and Agriculture Organization of the United Nations) Plant Protection Bulletin, 40(3), 89-94.

Lallemand, V. (1929a) Liste d'Homoptéres du Portugal recueillis par M. de Seabra. Memorias e Estudos do Museu Zoológico da Universidade de Coimbra, 28, 1-10.

Lallemand, V. (1929b) 2 2. Liste d'Homoptéres du Portugal recueillis par M. de Seabra. Memorias e Estudos do Museu Zoológico da Universidade de Coimbra, 39, 1-8.

Le Quesne, W.J. (1988) The Aphrodes bicinctus complex. In: Vidano, C.; Arzone, A. [Eds]. 6th Auchenorrhyncha Meeting, Turin, Italy, 7-11 ${ }^{\text {th }}$ September $1987,413-415$.

Leise, T. (1992) Zur Phylogenie der sudwest-Palaarktischen zikaden-Gattung Cyphopterum Melichar, 1905 (Homoptera: Auchenorrhyncha:Flatidae). Teil I-II: Abbildungen Dssertation zur erlangung des Doktorgrades der naturwissenschaften (Dr. rer. nat.). Lahn, 643pp.

Lin, L., Xu, Y., Liu, Y., Bao, S., Xin, Y. \& Li, X. (1996) Characteristics of Laodelphax striatellus transmit rice stripe virus. Acta Phytophylacica Sinica, 23(3), 218-222. 
Lindberg, H. (1936) Die Cicadinen der Kanarischen Inseln. Commentationes Biologicae, 4 (9), 1-19.

Lindberg, H. (1941) Die Hemipteren der Azorischen Inseln. Nebst anhang: Zur Kenntis der Hemipterenfauna von Madeira. Commentationes Biologicae, 8 (8), 1-32.

Lindberg, H. (1954) Hemiptera Insularum Canariensium, Systematik, Ökologie und Verbreitung der Kanarischen Heteropteren und Cicadinen. Commentationes Biologicae, 14 (1), 1953 (issued 1954), 1-304.

Lindberg, H. (1956) Beschreibung des neuen Issus maderensis (Hom., Issidae). Notulae Entomologicae, 36, 65-68.

Lindberg, H. (1959) A new species of the genus Cyphopterum Amyot (Hom., Flatidae) fom the Salvage Islands. Notulae Entomologicae, 39, 18-21.

Lindberg, H. (1960) Hemiptera from the Azores and Madeira. Boletim do Museu Municipal do Funchal, 13 (33), 85-94.

Lindberg, H. (1961) Hemiptera Insularum Madeirensium. Commentationes Biologicae, 24 (1), 1-82.

Lindberg, H. (1962) Weiterer beitrag zur kenntnis der Zikadenfauna Portugals. Notulae Entomologicae, 42, 24-26.

Lindberg, H. \& Wagner E. (1965) Supplementum secundum ad cognitionem Hemipterorum Insularum Canariensium. Commentationes Biologicae, 28 (10), 1-14.

Linnavuori, R. (1962) Hemiptera of Israel III. Annales Zoologici Societatis Vanamo, 24, 1-108.

Linnavuori, R. (1974) A hemipterous material from the Salvage Islands. Bocagiana, 34, 1-3.

Médail, F. \& Quézel, P. (1999) Biodiversity hotspots in the Mediterranean Basin: Setting global conservation priorities. Conservation Biology, 13 (6), 1510-1513.

Melichar, L. (1905) Genera tria fulgoridarum mundi antiqui. Annales Musei Nationalis Hungarici, 3, $473-477$.

Melichar, L. (1923) Homoptera, fam. Acanaloniidae, Flatidae et Ricaniidae. Genera Insectorum, 182, 1-185.

Müller, H.J. (1983) Uber den Einfluss extradianer Tageslangen auf das allometrische Wachstum von Euscelis incisus (Kbm) (Homoptera Auchenorrhyncha). 4. Beitrag zur weitere Analyse der Okomorphosen von Euscelis incisus. Zoologische Jahrbücher Abteilung für Systematik Öekologie und Geographie der Tiere, 110(3), 301-322.

Nast, J. (1972) Palaearctic Auchenorrhyncha (Homoptera). An annotated check list. Warszawa - Polish Scientific Publishers, $551 \mathrm{pp}$.

Nast, J. (1975) On some Mediterranean Delphacidae (Homoptera, Auchenorrhyncha) described by S. Matsumura. Annales Zoologici Warszawa, 33 (1), 1-15.

Nast, J. (1987) The Auchenorrhyncha (Homoptera) of Europe. Annales Zoologici, 40 (15), 535-661.

Noualhier, M. (1897) Hémiptères recueillis par M.A. Fauvel à Madère, en mai et juin 1896. Revue d'Entomologie, 16, 76-80.

Oromi, P., Aguin, D., Quartau, J.A. \& Zurita, N. (2004) Hemiptera Auchenorrhyncha. In: Izquierdo, I., J.L. Martín, N. Zurita \& Arechavaleta, M. (Eds.). Lista preliminar de especies silvestres de Canarias (hongos, plantas y animales terrestres). Consejería de Medio Ambiente y Ordenación Territorial, Gobierno de Canarias, pp. 198-207.

Palermo, S., Arzone, A. \& Bosco, D. (2001) Vector-pathogen-host plant relationships of chrysanthemum yellows (CY) phytoplasma and the vector leafhoppers Macrosteles quadripunctulatus and Euscelidius variegatus. Entomologia Experimentalis et Applicata, 99 (3), 347-354.

Press, J.R. \& Short, M.J. (1994) Flora of Madeira. The Natural History Museum, London, 574 pp.

Quartau, J.A. (1975) On the origin of two flatids (Homoptera Auchenorrhyncha) of the Salvage Islands. Bocagiana, 37, $1-8$.

Quartau, J.A. (1981) On a new species of the genus Brachypterona Lindberg (Homoptera: Cicadellidae) from the Salvage Islands. Arquivos do Museu Bocage, 1(10), 125-132.

Quartau, J.A. (1984) Two new records of leafhoppers (Homoptera, Auchenorryncha, Cicadellidae) from the Small Salvage Island. Bocagiana, 72, $1-7$.

Quartau, J.A. (1993) A preliminary biogeographic survey of the Madeiran leafhoppers (Homoptera, Auchenorryncha, Cicadellidae). Courier Forschungsinstitut Senckenberg, 159, 387-393.

Quartau, J.A. \& André, G. (1988) Neophilaenus angustipennis (Horváth, 1909) new to Madeira (Homoptera, Auchenorrhyncha, Cercopidae). Boletim do Museu Municipal do Funchal, 40 (206), 243-247.

Quartau, J.A. \& Fançony, A.I. (1988) On Eupteryx capreola Lindberg and E. filicum (Newman) (Homoptera: Cicadellidae, Typhlocybinae) from Madeira and the Azores. Boletim do Museu Municipal do Funchal, 40, 93-103.

Quartau, J.A. \& André, G. (1990) Peragallia caboverdensis Lindberg, 1958 new to the Salvages (Homoptera, Auchenorrhyncha, Cicadellidae). Boletim do Museu Municipal do Funchal, 42 (218), 67-70.

Quartau, J.A. \& Remane, R. (1996) Asianidia melliferae sp.n. (Homoptera: Auchenorrhyncha, Cicadellidae), a new leafhopper from Madeira. Bocagiana, 181, 1-3.

Quartau, J.A., Rodrigues, P.D. \& Marques, D. (2004) On the colour polymorphism of Asianidia chinai (Lindberg, 1961) in Madeira (Hemiptera: Cicadellidae, Typhlocybinae). Arquivos do Museu Bocage, 4 (2), 159-170.

Quartau, J.A. \& Borges, P. (2005) Hemiptera. Cicadomorpha, Fulgoromorpha. In: Borges, P., Cunha, R., Gabriel, R., Frias Martins, A., Silva, L. \& Vieira, V. (Eds.). A list of terrestrial fauna (Mollusca and Arthropoda) and flora (Briophyta, Pteridophyta and Spermatophyta) from Azores. Direcção Regional do Ambiente e Universidade dos Açores, Horta, Angra do Heroísmo e Ponta Delgada, pp. 190-191.

Razvyazkina, G.M. \& Pridantseva, E.A. (1968). Leafhoppers of the group Psammotettix striatus L. (Homoptera, Cicadel- 
lidae) - vectors of virus diseases of cereals, their systematics and distribution. Zoologicheskiy Zhurnal, 47(5), 690696.

Reis, F. \& Aguin-Pombo, D. (2003) Euscelidius variegatus (Kirschbaum, 1858), a new leafhopper record to Madeira Archipelago (Hemiptera, Cicadellidae). Vieraea, 21, 27-31.

Remane, R. (1968) Ergänzungen und kritische Bemerkungen zu der Heteropteren- und Cicadinen-Fauna der Makaronesischen Inseln. Bocagiana, 16, 1-15.

Remane, R. (1975). Eine bisher unbeschriebene Art der Gattung Javesella Fennah (Homopt. Cicadina: Delphacidae) von den Azoren. Stuttgarter Beiträge zur Naturkunde Serie A (Biologie), 269, 1-6.

Remane, R. (1985) Vorläufige anmerkungen zur Evolution und Speziation der Gattung Issus F. auf den Mittelatlantischen Inseln (Kanaren, Madeira) (Homoptera Auchenorrhyncha Fulgoromorpha Issidae). Marburger Entomologische Publikationen, 1 (10), 1-168.

Remane, R. \& Asche, M. (1979) Evolution und Speziation der gattung Cixius Latreille 1804 (Homoptera Auchenorrhyncha Fulgoromorpha Cixiidae) auf den Azorischen Inseln. Marburger Entomologische Publikationen, 1 (2), 1-264.

Remane, R. \& Asche, M. (1986) Makarorysa n.g. - new genus for the Canaro-Madeiran Eurysa ribauti Lindberg-group with remarks on speciation, distribution and phylogenetic relationships within this taxon (Homoptera, Auchenorrhyncha, Fulgoromorpha, Delphacidae). Marburger Entomologische Publikationen, 2 (3), 217-258.

Remane, R. \& Hoch, H. (1986) Sechs neue Arten der gattung Hyalesthes Signoret, 1865 (Homoptera, Fulgoroidea, Cixiidae) von den Mittelatlantischen Inseln und aus dem Irak. Marburger Entomologische Publikationen, 2 (3), 123154.

Schmidt, E. (1915) Die Dictyopharinen des Stettiner Museum (Hemiptera - Homoptera). Stettiner Entomologische Zeitung, 76, 345-358.

Seabra, A.F. (1930a) Observações sobre algumas espécies da Fam. Cicadoideae e da Fam. Fulgoroideae existentes nas colecções do Museu de Coimbra. Museu Zoológico da Universidade de Coimbra, Arquivos da Secção de Biologia e Parasitologia, 1, 307-359.

Seabra, A.F. (1930b) Subsidio para o conhecimento da fauna das Matas Nacionais. Conclusões de estudos realizados durante os meses de Julho e Agosto de 1925 na Mata de Leiria. Museu Zoológico da Universidade de Coimbra. Arquivos da Secção de Biologia e Parasitologia, 1, 215-260.

Seabra, A.F. (1939a) A entomologia do trigo. Museu Zoológico da Universidade de Coimbra, Arquivos da Secção de Biologia e Parasitologia, 3, 84-93.

Seabra, A.F. (1939b) Contribuição para a historia da Entomologia em Portugal. Direcção Geral dos Serviços Florestais e Agricolas, 6 (1-2), 1-301.

Seabra, A.F. (1941) Contribuições para o inventário da fauna lusitânia. Insecta. Homoptera (Cicadoideae e Fulgororideae). Memorias e Estudos do Museu Zoológico da Universidade de Coimbra, 121, 1-14.

Vitousek, P.M. (1988) Diversity and biological invasions of oceanic islands. In: E. O. Wilson (Ed.), Biodiversity. National Academy of Sciences, U.S.A. pp. 181-189.

Waldén, H.W. (1983) Systematic and biogeographical studies in the terrestrial Gastropoda of Madeira with an annotated check-list. Annales Zoologici Fennici, 20, 255-275.

Walker, F. (1851) List of the specimens of homopterous insects in the collection of the British Museum Part II. London, pp. 262-636.

Webb, M. (1987) Species recognition in Cicadulina leafhoppers (Hemiptera: Cicadellidae), vectors of pathogens of Gramineae. Bulletin of Entomological Research, 77, 683-712.

Webb, M. D. \& Viraktamath, C. A. (2004). On the identity of an invasive leafhopper on Hawaii (Hemiptera, Cicadellidae, Nirvaninae). Zootaxa. 692: 1-6.

Wetterer, J.K., Espadaler, X., Wetterer, A. L., Aguin-Pombo, D. \& Franquinho-Aguiar, A.M. (2006) Long-term impact of exotic ants on the native ants of Madeira. Ecological Entomology, 31 (4), 358-368.

Young, D.A. \& Frazier, N.W. (1954) A study of the leafhopper genus Circulifer Zakhvatkin (Homoptera, Cicadellidae). Hilgardia, 23 (2), 25-57. 